\title{
A RENORMALIZED NEWTON METHOD FOR LIQUID CRYSTAL DIRECTOR MODELING
}

\author{
EUGENE C. GARTLAND, JR* AND ALISON RAMAGE ${ }^{\dagger}$
}

\begin{abstract}
We consider the nonlinear systems of equations that result from discretizations of a prototype variational model for the equilibrium director field characterizing the orientational properties of a liquid crystal material. In the presence of pointwise unit-vector constraints and coupled electric fields, the numerical solution of such equations by Lagrange-Newton methods leads to linear systems with a double saddle-point form, for which we have previously proposed a preconditioned nullspace method as an effective solver [A. Ramage and E. C. Gartland, Jr., SIAM J. Sci. Comput., 35 (2013), pp. B226-B247]. Here we propose and analyze a modified outer iteration ("Renormalized Newton Method") in which the orientation variables are normalized onto the constraint manifold at each iterative step. This scheme takes advantage of the special structure of these problems, and we prove that it is locally quadratically convergent. The Renormalized Newton Method bears some resemblance to the Truncated Newton Method of computational micromagnetics, and we compare and contrast the two. This brings to light some anomalies of the Truncated Newton Method.
\end{abstract}

Key words. liquid crystals, director models, unit-vector constraints, saddle-point problems, reduced Hessian method, Renormalized Newton Method

AMS subject classifications. 49K35, 49M15, 65H10, 65K10, 65N22

1. Introduction. Many continuum models for the orientational properties of liquid crystals involve one or more state variables that are vector fields of unit length. The pointwise unit-vector constraints associated with discretizations of equilibrium models of such systems give rise to indefinite linear algebraic equations of saddle-point form when these constraints are imposed via Lagrange multipliers. In problems such as these, indefiniteness also frequently manifests itself due to another influence, coupling with applied electric fields, and this leads to a double saddle-point structure. Models with some similar features arise also in the area of computational micromagnetics.

In [21] we analyzed a model problem of this type and proposed a nullspace method using MINRES with diagonal block preconditioning as a natural approach to solve the linear systems that result when Newton's method is applied to the Lagrangian. The main ideas are briefly summarized below. These models are nonlinear and depend on multiple physical and geometric parameters, and it is typical for the equilibrium solutions (phases) to undergo transitions at critical values of certain of these parameters. The context we imagine is the numerical bifurcation and phase analysis of a discretization of a model for a realistic device or experiment in the large scale regime - this is the main motivation for this work. In such a setting, parameter continuation leads to the repeated solution of systems of the type we are studying here. In the course of such path following, good initial guesses are available, however, and global Newton methods are generally employed.

At each computed equilibrium point along a branch of solutions, one performs auxiliary calculations of the free energy and the local stability of the solution - it is necessary to be able to compute both stable and unstable solutions. For parameter ranges in which multiple equilibrium solutions exist, the solution of least free energy

\footnotetext{
${ }^{*}$ Department of Mathematical Sciences, Kent State University, Kent, Ohio 44242 USA (gartland@math.kent.edu). Research supported in part by the National Science Foundation grant DMS-1211597.

${ }^{\dagger}$ Department of Mathematics and Statistics, University of Strathclyde, Glasgow G1 1XH, Scotland (A.Ramage@strath.ac.uk).
} 
gives the globally stable phase of the system. Local stability is characterized by certain eigenvalue calculations and identifies equilibria that have the potential of being globally stable. Aspects of this are discussed in [9] and [10].

The objectives of this present paper are to introduce and analyze an alternative outer iteration ("Renormalized Newton Method"), which is a variation of Newton's method that takes greater advantage of the special structure of such problems and which we prove retains the local quadratic convergence properties of Newton's method. Also presented are results of numerical experiments comparing the performance of the basic Newton Method and the Renormalized Newton Method on a model problem that admits multiple distinct solutions, one with a defect (singularity) and two others without. In addition, comparisons are made between the Renormalized Newton Method and the "Truncated Newton Method" of computational micromagnetics [8, $\S 4.2]$, for which we also provide some analytical observations. The separate document of Supplementary Material contains more background on the liquid crystal model (including its relation to the Landau-Lifshitz model of ferromagnetics), demonstration of the lack of an "energy decay property" for general liquid crystal free energy functionals (under renormalizing a director field of greater than unit length), and results of some numerical experiments on the Truncated Newton Method.

2. A prototype liquid crystal director model with a coupled electric field. Many experiments and devices involving liquid crystal materials can be effectively modeled using a macroscopic continuum framework in which the orientational state of the system is described by a director field (a unit-length vector field representing the average orientation of the molecules in a fluid element at a point), traditionally denoted by $\boldsymbol{n}$. Most devices and many experiments involve the interaction between a liquid crystal material and an applied electric field (which is used to control the liquid crystal orientational properties). This is a coupled interaction, with the electric field influencing the orientations of the molecules and the molecular orientational properties in turn influencing the local electric fields through their effect on the dielectric tensor. The free energy is the thermodynamic potential that determines equilibrium states in systems such as these, for which we consider the simplest prototype model:

$$
\mathcal{F}[\boldsymbol{n}, U]=\frac{1}{2} \int_{\Omega}\left[K|\nabla \boldsymbol{n}|^{2}-\varepsilon(\boldsymbol{n}) \nabla U \cdot \nabla U\right], \quad|\nabla \boldsymbol{n}|^{2}=\sum_{i, j=1}^{3}\left(\frac{\partial n_{i}}{\partial x_{j}}\right)^{2} .
$$

Here $\Omega$ is the region occupied by the liquid crystal material, $K$ is a positive materialdependent "elastic constant," $\varepsilon$ is the dielectric tensor (which depends on $\boldsymbol{n}$ ), and $U$ is the electrostatic potential. The local electric field is given by $\boldsymbol{E}=-\nabla U$, and the expression above for $|\nabla \boldsymbol{n}|^{2}$ is for a fixed Cartesian frame. See [22, §2.2], [23, §3.2], and the Supplementary Material for more detail.

The tensor field $\varepsilon$ is real, symmetric, and positive definite, with eigenvalues $\varepsilon_{0} \varepsilon_{\|}$ (for eigenvectors parallel to $\boldsymbol{n}$ ) and $\varepsilon_{0} \varepsilon_{\perp}$ (for vectors perpendicular to $\boldsymbol{n}$ ). Here $\varepsilon_{0}$ is the vacuum dielectric constant, and $\varepsilon_{\|}$and $\varepsilon_{\perp}$ are the relative permittivities of the material. The parameters $\varepsilon_{0}, \varepsilon_{\|}$, and $\varepsilon_{\perp}$ are all positive, while the "dielectric anisotropy" $\varepsilon_{\mathrm{a}}:=\varepsilon_{\|}-\varepsilon_{\perp}$ can be positive or negative (and gives rise to a torque on the molecules due to the electric field). The strong form of the constrained equilibrium equations for (2.1) is

$$
-K \Delta \boldsymbol{n}=\lambda \boldsymbol{n}+\varepsilon_{0} \varepsilon_{\mathrm{a}}(\nabla U \cdot \boldsymbol{n}) \nabla U, \quad \operatorname{div}(\varepsilon(\boldsymbol{n}) \nabla U)=0, \quad|\boldsymbol{n}|=1,
$$

which is to be solved in $\Omega$ subject to appropriate boundary conditions on $\boldsymbol{n}$ and $U$. Here the Lagrange multiplier field $\lambda$ is associated with the pointwise unit-vector 
constraint, and the electrostatics equation takes the form

$$
\operatorname{div}(\varepsilon(\boldsymbol{n}) \nabla U)=\sum_{i, j} \frac{\partial}{\partial x_{i}}\left(\varepsilon_{i j} \frac{\partial U}{\partial x_{j}}\right)=0
$$

with respect to a fixed Cartesian frame.

A distinguishing feature of these problems is the intrinsic saddle-point nature of the electric-field coupling: equilibria are minimizing with respect to $\boldsymbol{n}$ but maximizing with respect to $U$. These models bear some similarity to the Landau-Lifshitz free-energy model for equilibrium states of magnetization in ferromagnetic materials, and we carefully compare and contrast these in $\S \mathrm{S} 1.2$ of the Supplementary Material. It is in general convenient for analysis and appropriate for numerical explorations to express all aspects of the problem (free-energy functional, Euler-Lagrange equations, etc.) in dimensionless form, and we give a typical non-dimensionalization in $\S S 1.3$ of the Supplementary Material. Under such a rescaling, the basic forms of all the expressions remains the same, and so we will retain the notation above. From this point on, however, we assume that all quantities are dimensionless, following a reasonable non-dimensionalization such as that in $\S S 1.3$.

3. Lagrange-Newton scheme and nullspace method. One can discretize a coupled, constrained equilibrium problem of the type presented in $\S 2$ in a variety of ways, starting from weak or strong formulations of (2.2) and utilizing various types of finite elements or finite differences or other discretization methods. Our preference is to approximate the free energy functional $\mathcal{F}$ directly by some appropriate finite elements and quadrature scheme, obtaining

$$
\mathcal{F}[\boldsymbol{n}, U] \approx f(\mathbf{n}, \mathbf{U}), \quad \mathbf{n}=\left(\mathbf{n}_{1}, \ldots, \mathbf{n}_{n}\right), \quad \mathbf{n}_{j} \in \mathbb{R}^{3}, \quad \mathbf{U}=\left(U_{1}, \ldots, U_{n}\right),
$$

where $\mathbf{n}$ and $\mathbf{U}$ contain the discrete director and electric potential degrees of freedom in some ordering. Here $n$ represents the total number of free nodes in the discrete model. The precise details of the discretization, which can be in any number of space dimensions, are not important. The unit-length constraint is to be imposed on the local director at each free node. This can be done using either Lagrange multipliers or penalty methods, for example. Our preference is for the former, because of issues related to conditioning, choosing penalty parameters, and the like. Augmented Lagrangian methods have also been suggested for such problems - see [12, §3.7].

In our approach, the full set of discrete, coupled, equality constrained equilibrium equations derives from a Lagrangian:

$$
\nabla L=\mathbf{0}, \quad \text { where } L(\mathbf{n}, \boldsymbol{\lambda}, \mathbf{U})=f(\mathbf{n}, \mathbf{U})+\sum_{j=1}^{n} \lambda_{j} g_{j}(\mathbf{n}), \quad g_{j}(\mathbf{n}):=\frac{1}{2}\left(\left|\mathbf{n}_{j}\right|^{2}-1\right) .
$$

Here $\boldsymbol{\lambda}=\left(\lambda_{1}, \ldots, \lambda_{n}\right)$ is the vector of Lagrange multipliers. In most circumstances, the system (3.1) can be seen to be a consistent approximation to (2.2) (in an appropriate scaling) - see [21] and $\S 5.1$ below for specific examples. The same basic idea can be used for problems in any number of space dimensions. In one, two, or three dimensions, the single, double, or triple integrals defining $\mathcal{F}$ would give rise to discretized equilibrium equations (and Hessian blocks below) that scale differently with respect to mesh parameters. While such aspects have the potential to affect condition numbers and scaling strategies (which will be addressed in [10]), they are not of concern for the analysis that follows, which is for a discretized problem on a fixed grid. 
3.1. Newton equations. A global Newton method applied to the system $\nabla L=$ $\mathbf{0}$ in (3.1) above leads to a linear system for the Newton corrections of the form

$$
\left[\begin{array}{ccc}
A & B & D \\
B^{T} & O & O \\
D^{T} & O & -C
\end{array}\right]\left[\begin{array}{l}
\boldsymbol{\delta} \mathbf{n} \\
\boldsymbol{\delta} \boldsymbol{\lambda} \\
\boldsymbol{\delta} \mathbf{U}
\end{array}\right]=-\left[\begin{array}{c}
\nabla_{\mathbf{n}} L \\
\nabla_{\boldsymbol{\lambda}} L \\
\nabla_{\mathbf{U}} L
\end{array}\right]
$$

where

$$
A=\nabla_{\mathbf{n n}}^{2} L, \quad B=\nabla_{\mathbf{n} \boldsymbol{\lambda}}^{2} L, \quad D=\nabla_{\mathbf{n} \mathbf{U}}^{2} L, \quad C=-\nabla_{\mathbf{U} \mathbf{U}}^{2} L,
$$

and $O$ denotes a zero matrix of appropriate dimensions. Our main interest is in effective numerical bifurcation and phase exploration of problems with such structure in the large scale regime, where iterative methods are called for, and where one must be able to compute both stable and unstable solutions. It is important to appreciate that the $A$ matrix block in (3.2) need not be positive definite or even nonsingular - this is demonstrated in the numerical experiments in $\S 5$ below.

For a particular application, the specific form of $f(\mathbf{n}, \mathbf{U})$ in (3.1) depends upon the form of $\mathcal{F}[\boldsymbol{n}, U]$ and on the details of the discretization used. Thus the composition of the Hessian matrix is somewhat problem dependent. Exact Hessians (not approximated ones) are used in general, and in [21] the structure of the matrix blocks in (3.2) is described in detail for a specific model problem discretized via piecewiselinear finite elements. There are some features of the Hessian matrix that are common to all problems. Observe that

$$
L=f+\sum_{j=1}^{n} \lambda_{j} g_{j} \Rightarrow \nabla_{\mathbf{n}} L=\nabla_{\mathbf{n}} f+\sum_{j=1}^{n} \lambda_{j} \nabla_{\mathbf{n}} g_{j}
$$

with $g_{j}=\frac{1}{2}\left(\left|\mathbf{n}_{j}\right|^{2}-1\right)$, from which follows

$$
\nabla_{\mathbf{n}} g_{j}=\left[\mathbf{0}, \ldots, \mathbf{0}, \mathbf{n}_{j}, \mathbf{0}, \ldots, \mathbf{0}\right]^{T} \text { and } \nabla_{\mathbf{n n}}^{2} g_{j}=\operatorname{diag}(O, \ldots, O, I, O, \ldots, O) .
$$

Here $\mathbf{n}_{j}$ is in the $j$-th position in the column vector $\nabla_{\mathbf{n}} g_{j}$ (and the zero vectors are 3 -vectors), and $I$ is in the $(j, j)$ position in $\nabla_{\mathbf{n n}}^{2} g_{j}$ (and the zero matrices and identity matrix are $3 \times 3$ ). We see that the $A$ matrix is $3 n \times 3 n$ and has the general form

$$
A=\nabla_{\mathbf{n n}}^{2} L=A_{0}+\Lambda, \quad A_{0}=\nabla_{\mathbf{n n}}^{2} f, \quad \Lambda=\left[\begin{array}{ccc}
\Lambda_{1} & & \\
& \ddots & \\
& & \Lambda_{n}
\end{array}\right], \Lambda_{j}=\left[\begin{array}{lll}
\lambda_{j} & & \\
& \lambda_{j} & \\
& & \lambda_{j}
\end{array}\right] \text {. }
$$

For our model problem (2.1), the leading terms of $A_{0}$ would resemble a discretization of $-K \Delta \boldsymbol{n}$ (in a certain scaling), although lower-order terms could cause loss of positive definiteness. We also see that the $B$ matrix is $3 n \times n$ and is given by

$$
B=\nabla_{\mathbf{n} \boldsymbol{\lambda}}^{2} L=\left[\nabla_{\mathbf{n}} g_{1}, \ldots, \nabla_{\mathbf{n}} g_{n}\right]=\left[\begin{array}{ccc}
\mathbf{n}_{1} & & \\
& \ddots & \\
& & \mathbf{n}_{n}
\end{array}\right] .
$$

Under a reasonable discretization, the $n \times n$ matrix $C$ will be real, symmetric, and positive definite, corresponding to the coefficient matrix associated with a discretization of (2.3). The matrix $D$ is $3 n \times n$ and embodies the coupling between $\delta \mathbf{n}$ and $\boldsymbol{\delta} \mathbf{U}$. See $[21, \S 3.3]$ or $\S 5.1$ below for specific examples. 
A point to keep in mind is that in general, the matrix $A$ depends on all of $\mathbf{n}, \mathbf{U}$, and $\lambda$ :

$$
A=A(\mathbf{n}, \boldsymbol{\lambda}, \mathbf{U})=A_{0}(\mathbf{n}, \mathbf{U})+\Lambda(\boldsymbol{\lambda}) .
$$

For our simplified model problem (2.1), though, $A_{0}$ (and hence $A$ ) is independent of n. The positive definiteness of $C$ generally derives from the uniform ellipticity of the electric potential terms in $\mathcal{F}$ (see the Supplementary Material). The specific details of the confinement of the sample and the boundary conditions on $U$ play a role, and these vary from problem to problem.

At regular solution points (away from bifurcation points and turning points), the coefficient matrix is symmetric, non-singular, and indefinite - that is, it has both positive and negative eigenvalues - and the linear system is in so-called saddle point form. Note that when both pointwise unit-vector constraints and coupled electric fields are present, these problems have a double saddle-point structure, as seen in (3.2). For a model with no electric field or for one with no unit-vector constraints (for example, if one were able to use an angle representation for $\boldsymbol{n}$ ), one would obtain instead either of the more common saddle-point forms

$$
\left[\begin{array}{cc}
A & B \\
B^{T} & O
\end{array}\right] \text { or } \quad\left[\begin{array}{cc}
A & D \\
D^{T} & -C
\end{array}\right] \text {. }
$$

3.2. Nullspace method. The nullspace method (or reduced Hessian method) is a technique for eliminating constraint blocks in systems such as these ((3.2) or systems with a coefficient matrix like the left side above), by using a matrix, which we denote $Z$, whose column space is the null space of $B^{T}$ - see for example $[2, \S 6]$. As we have shown in [21], it is trivial to construct such a matrix for problems such as ours, and it takes the form

$$
Z=\left[\begin{array}{lllllll}
\mathbf{l}_{1} & \mathbf{m}_{1} & & & & & \\
& & \mathbf{l}_{2} & \mathbf{m}_{2} & & & \\
& & & & \ddots & & \\
& & & & & \mathbf{l}_{n} & \mathbf{m}_{n}
\end{array}\right] .
$$

Here $\mathbf{l}_{j}$ and $\mathbf{m}_{j}$ are local 3-vectors and are constructed by simple formulas from $\mathbf{n}_{j}$ such that, at each node, $\mathbf{l}_{j}, \mathbf{m}_{j}, \mathbf{n}_{j}$ form an orthogonal triple, and we assume that $\mathbf{n}_{j} \neq \mathbf{0}, \forall j$ (the "non-degeneracy condition" of [21]).

Consider now the second block equation of (3.2),

$$
B^{T} \boldsymbol{\delta} \mathbf{n}=-\nabla_{\boldsymbol{\lambda}} L
$$

which is an under-determined system of $n$ equations in $3 n$ unknowns. Assuming nondegeneracy of the current local directors $\left(\mathbf{n}_{j} \neq \mathbf{0}, \forall j\right)$, the columns of both $B$ and $Z$ are linearly independent and together form an orthogonal basis for $\mathbb{R}^{3 n}$, as a result of which we have

$$
B^{T} Z=O_{n \times 2 n}, \quad Z^{T} B=O_{2 n \times n}, \quad B^{T} B, Z^{T} Z \text { diagonal and nonsingular, }
$$

and (3.6) is guaranteed to be consistent. Decomposing a solution of (3.6) into its components in the normal space to the constraint manifold at $\mathbf{n}$ and those in the tangent space,

$$
\delta \mathbf{n}=B \mathbf{r}+Z \mathbf{p}, \quad \mathbf{r} \in \mathbb{R}^{n}, \quad \mathbf{p} \in \mathbb{R}^{2 n}
$$


and substituting this decomposition into (3.6), one obtains

$$
B^{T} \boldsymbol{\delta} \mathbf{n}=B^{T} B \mathbf{r}+B^{T} Z \mathbf{p}=B^{T} B \mathbf{r}=-\nabla_{\boldsymbol{\lambda}} L \Rightarrow \mathbf{r}=-\left(B^{T} B\right)^{-1} \nabla_{\boldsymbol{\lambda}} L,
$$

and we see that the solution set of (3.6) can be written

$$
\boldsymbol{\delta} \mathbf{n}=\widehat{\boldsymbol{\delta} \mathbf{n}}+Z \mathbf{p}, \quad \widehat{\boldsymbol{\delta} \mathbf{n}}:=-B\left(B^{T} B\right)^{-1} \nabla_{\boldsymbol{\lambda}} L, \quad \mathbf{p} \text { arbitrary }
$$

Projecting the first block equation of (3.2) into its normal-space and tangentspace components gives

$$
\begin{aligned}
& B^{T} A \boldsymbol{\delta} \mathbf{n}+B^{T} B \boldsymbol{\delta} \boldsymbol{\lambda}+B^{T} D \boldsymbol{\delta} \mathbf{U}=-B^{T} \nabla_{\mathbf{n}} L \\
& Z^{T} A \boldsymbol{\delta} \mathbf{n}+Z^{T} B \boldsymbol{\delta} \boldsymbol{\lambda}+Z^{T} D \boldsymbol{\delta} \mathbf{U}=-Z^{T} \nabla_{\mathbf{n}} L .
\end{aligned}
$$

The first equation above can be solved for $\boldsymbol{\delta} \boldsymbol{\lambda}$ once $\boldsymbol{\delta} \mathbf{n}$ and $\boldsymbol{\delta} \mathbf{U}$ have been determined:

$$
\boldsymbol{\delta} \boldsymbol{\lambda}=-\left(B^{T} B\right)^{-1} B^{T}\left(\nabla_{\mathbf{n}} L+A \boldsymbol{\delta} \mathbf{n}+D \boldsymbol{\delta} \mathbf{U}\right) .
$$

Substituting the representation (3.7) into the second equation (and using $Z^{T} B=O$ ) gives

$$
Z^{T} A Z \mathbf{p}+Z^{T} D \delta \mathbf{U}=-Z^{T} \nabla_{\mathbf{n}} L-Z^{T} A \widehat{\boldsymbol{\delta} \mathbf{n}}
$$

while using the same representation $\delta \mathbf{n}=\widehat{\boldsymbol{\delta n}}+Z \mathbf{p}$ in the third block equation of (3.2) gives

$$
D^{T} \boldsymbol{\delta} \mathbf{n}-C \boldsymbol{\delta} \mathbf{U}=-\nabla_{\mathbf{U}} L \Rightarrow D^{T} Z \mathbf{p}-C \boldsymbol{\delta} \mathbf{U}=-\nabla_{\mathbf{U}} L-D^{T} \widehat{\boldsymbol{\delta} \mathbf{n}} .
$$

The equations for $\mathbf{p}$ and $\boldsymbol{\delta} \mathbf{U}$ (uncoupled now from the equations for $\boldsymbol{\delta} \boldsymbol{\lambda}$ ) can thus be written as a reduced $3 n \times 3 n$ system:

$$
\left[\begin{array}{cc}
Z^{T} A Z & Z^{T} D \\
D^{T} Z & -C
\end{array}\right]\left[\begin{array}{c}
\mathbf{p} \\
\boldsymbol{\delta} \mathbf{U}
\end{array}\right]=-\left[\begin{array}{c}
Z^{T}\left(\nabla_{\mathbf{n}} L+A \widehat{\boldsymbol{\delta} \mathbf{n}}\right) \\
\nabla_{\mathbf{U}} L+D^{T} \widehat{\boldsymbol{\delta} \mathbf{n}}
\end{array}\right]
$$

Note again that $B^{T} B$ is a diagonal matrix, and this is true in any number of space dimensions - once a consistent ordering of the nodes and constraints is decided upon, the $B$ matrix always has the generic form (3.4). Thus the computation of $\widehat{\boldsymbol{\delta} \mathbf{n}}$ and $\boldsymbol{\delta} \boldsymbol{\lambda}$ is quite simple. This approach is examined analytically and through numerical experiments in [21] on a specific model problem.

3.3. Geometric interpretation. The particular solution $\widehat{\boldsymbol{\delta n}}$ in (3.7) can be seen to be the minimum 2-norm solution of $B^{T} \boldsymbol{\delta} \mathbf{n}=-\nabla_{\boldsymbol{\lambda}} L$ (since $\|\boldsymbol{\delta} \mathbf{n}\|_{2}^{2}=\|\widehat{\boldsymbol{\delta} \mathbf{n}}\|_{2}^{2}+$ $\|Z \mathbf{p}\|_{2}^{2}$ in (3.7), by virtue of the mutual orthogonality of the columns of $B$ and those of $Z$ ). Pointwise it has the explicit form

$$
(\widehat{\boldsymbol{\delta} \mathbf{n}})_{j}=\frac{1}{2}\left(\frac{1-\left|\mathbf{n}_{j}\right|^{2}}{\left|\mathbf{n}_{j}\right|^{2}}\right) \mathbf{n}_{j}, \quad j=1, \ldots, n
$$

Thus the representation (3.7) decomposes the increment $\boldsymbol{\delta} \mathbf{n}$ at each point into a component parallel to $\mathbf{n}_{j}\left((\widehat{\boldsymbol{\delta} \mathbf{n}})_{j}\right.$ above $)$ and a component perpendicular to $\mathbf{n}_{j}\left((Z \mathbf{p})_{j}=\right.$ $p_{j} \mathbf{l}_{j}+q_{j} \mathbf{m}_{j}$, where $\left.\mathbf{p}=\left[p_{1}, q_{1}, \ldots, p_{n}, q_{n}\right]^{T}\right)$. The component $(\widehat{\boldsymbol{\delta} \mathbf{n}})_{j}$ is local, completely driven by the pointwise unit-vector constraint, and independent of the liquid 
crystal distortional elasticity (which is captured by the components of $Z \mathbf{p}$ ). It can be seen as a linearized correction in the $\mathbf{n}_{j}$ direction towards satisfying the unit-vector constraint at the $j$-th grid point.

One can compare $(\widehat{\boldsymbol{\delta} \mathbf{n}})_{j}$ in (3.10) with the true increment (in the $\mathbf{n}_{j}$ direction) that would be needed to bring an un-normalized local director $\mathbf{n}_{j}$ onto the local constraint manifold $\left|\mathbf{n}_{j}\right|=1$ :

$$
\mathbf{n}_{j} \mapsto \frac{\mathbf{n}_{j}}{\left|\mathbf{n}_{j}\right|}=\mathbf{n}_{j}+\delta \mathbf{n}_{j}^{\text {true }} \Rightarrow \delta \mathbf{n}_{j}^{\text {true }}=\frac{1-\left|\mathbf{n}_{j}\right|}{\left|\mathbf{n}_{j}\right|} \mathbf{n}_{j}=\frac{2\left|\mathbf{n}_{j}\right|}{1+\left|\mathbf{n}_{j}\right|}(\widehat{\delta \mathbf{n}})_{j}
$$

Since

$$
\frac{2\left|\mathbf{n}_{j}\right|}{1+\left|\mathbf{n}_{j}\right|}>1 \Leftrightarrow\left|\mathbf{n}_{j}\right|>1 \quad \text { and } \quad \frac{2\left|\mathbf{n}_{j}\right|}{1+\left|\mathbf{n}_{j}\right|}<1 \Leftrightarrow\left|\mathbf{n}_{j}\right|<1
$$

we see that $(\widehat{\boldsymbol{\delta n}})_{j}$ is too large if $\left|\mathbf{n}_{j}\right|<1$ and too small if $\left|\mathbf{n}_{j}\right|>1$. We conclude that the calculated Newton correction $\boldsymbol{\delta} \mathbf{n}=\widehat{\boldsymbol{\delta} \mathbf{n}}+Z \mathbf{p}$ necessarily produces a new local director

$$
\mathbf{n}_{j}+\boldsymbol{\delta} \mathbf{n}_{j}=\mathbf{n}_{j}+(\widehat{\boldsymbol{\delta} \mathbf{n}})_{j}+(Z \mathbf{p})_{j}, \quad(\widehat{\boldsymbol{\delta} \mathbf{n}})_{j} \| \mathbf{n}_{j}, \quad(Z \mathbf{p})_{j} \perp \mathbf{n}_{j}
$$

that satisfies

$$
\left|\mathbf{n}_{j}+\delta \mathbf{n}_{j}\right| \geq 1
$$

with equality above if and only if $\left|\mathbf{n}_{j}\right|=1$ (which implies that $(\widehat{\boldsymbol{\delta} \mathbf{n}})_{j}=\mathbf{0}$ ) and $(Z \mathbf{p})_{j}=\mathbf{0}$. Thus successive Newton iterates generally exceed the pointwise unitvector normalization, approaching it in the limit as the Newton iteration converges. We will use these observations in an attempt to accelerate this process.

4. Alternative outer iteration: Renormalized Newton Method. While a global Newton scheme is a natural choice for an outer iteration, specific features of problems such as these (with such pointwise unit-vector constraints) suggest some simplifications, which lead to a closely related variant. In particular, we have already observed the simplifications that accompany the circumstance in which the current approximate $\mathbf{n}$ is normalized: $\left|\mathbf{n}_{j}\right|=1, j=1, \ldots, n$. In this situation, $\mathbf{l}_{j}, \mathbf{m}_{j}, \mathbf{n}_{j}$ form an orthonormal triple at each grid point, and $B^{T} B=I$. Since $\nabla_{\boldsymbol{\lambda}} L=\mathbf{0}, \widehat{\boldsymbol{\delta} \mathbf{n}}=\mathbf{0}$, and $\delta \mathbf{n}_{j} \perp \mathbf{n}_{j}$ for all $j$, the nullspace-method equations (3.9) and (3.8) take the simpler form

$$
\begin{gathered}
{\left[\begin{array}{cc}
Z^{T} A Z & Z^{T} D \\
D^{T} Z & -C
\end{array}\right]\left[\begin{array}{c}
\mathbf{p} \\
\boldsymbol{\delta} \mathbf{U}
\end{array}\right]=-\left[\begin{array}{c}
Z^{T} \nabla_{\mathbf{n}} f \\
\nabla_{\mathbf{U}} f
\end{array}\right], \quad \boldsymbol{\delta} \mathbf{n}=Z \mathbf{p}} \\
\boldsymbol{\delta} \boldsymbol{\lambda}=-B^{T}\left(\nabla_{\mathbf{n}} f+A \boldsymbol{\delta} \mathbf{n}+D \boldsymbol{\delta} \mathbf{U}\right)
\end{gathered}
$$

Here we have used the facts that

$$
\nabla_{\mathbf{n}} L=\nabla_{\mathbf{n}} f+B \boldsymbol{\lambda} \Rightarrow Z^{T} \nabla_{\mathbf{n}} L=Z^{T} \nabla_{\mathbf{n}} f
$$

since $Z^{T} B=O$ by construction, and $\nabla_{\mathbf{U}} L=\nabla_{\mathbf{U}} f$. By virtue of the simple, local nature of our constraints, it is quite easy to force them upon any approximate discrete director field $\mathbf{n}$ by simply normalizing each local director. Furthermore, we know from the discussion in $\S 3.3$ that the basic Newton iteration produces local directors that are systematically too long $\left(\left|\mathbf{n}_{j}\right|>1\right)$. 
Also, the Lagrange multipliers occur in a simple way, linearly in the $\nabla_{\mathbf{n}} L=\mathbf{0}$ equations, and in general these are not of the same level of physical interest as are $\mathbf{n}$ and $\mathbf{U}$. Given the $\mathbf{n}^{*}$ and $\mathbf{U}^{*}$ components of an exact solution of $\nabla L\left(\mathbf{n}^{*}, \boldsymbol{\lambda}^{*}, \mathbf{U}^{*}\right)=\mathbf{0}$, the Lagrange multipliers can be computed directly (and locally) via

$$
\begin{gathered}
\nabla_{\mathbf{n}} L=\nabla_{\mathbf{n}} f+B \boldsymbol{\lambda}=\mathbf{0} \Rightarrow \boldsymbol{\lambda}^{*}=-B\left(\mathbf{n}^{*}\right)^{T} \nabla_{\mathbf{n}} f\left(\mathbf{n}^{*}, \mathbf{U}^{*}\right) \\
\Leftrightarrow \quad \lambda_{j}^{*}=-\nabla_{\mathbf{n}_{j}} f\left(\mathbf{n}^{*}, \mathbf{U}^{*}\right) \cdot \mathbf{n}_{j}^{*}, \quad j=1, \ldots, n .
\end{gathered}
$$

When $\mathbf{n}$ and $\mathbf{U}$ correspond to the components of an approximate (not yet converged) solution of $\nabla L=\mathbf{0}$, the over-determined system $\nabla_{\mathbf{n}} f+B \boldsymbol{\lambda}=\mathbf{0}$ (viewed as $3 n$ equations in $\lambda_{1}, \ldots, \lambda_{n}$ ) is not necessarily consistent, and the formulas above give the linear least squares solution:

$$
\min _{\boldsymbol{\lambda}}\left\|\nabla_{\mathbf{n}} f+B \boldsymbol{\lambda}\right\|_{2} \quad \Leftrightarrow \quad \boldsymbol{\lambda}=-\left(B^{T} B\right)^{-1} B^{T} \nabla_{\mathbf{n}} f=-B^{T} \nabla_{\mathbf{n}} f
$$

These then are the features we will exploit: renormalizing the discrete director at each outer iterative step and eliminating the Lagrange multipliers by the formulas above. Our algorithm for the "Renormalized Newton Method" takes the following form.

Algorithm 4.1 (Renormalized Newton Method). Repeat until convergence:

1. in: $\mathbf{n}$, U satisfying $\left|\mathbf{n}_{j}\right|=1, j=1, \ldots, n$

2. build $B(\mathbf{n})$ and $\nabla_{\mathbf{n}} f(\mathbf{n}, \mathbf{U})$

3. set

$$
\lambda=-B^{T} \nabla_{\mathbf{n}} f
$$

4. build $A(\mathbf{n}, \boldsymbol{\lambda}, \mathbf{U})=A_{0}(\mathbf{n}, \mathbf{U})+\Lambda(\boldsymbol{\lambda}), C(\mathbf{n}), D(\mathbf{n}, \mathbf{U}), Z(\mathbf{n})$, and $\nabla_{\mathbf{U}} f(\mathbf{n}, \mathbf{U})$

5. solve

$$
\left[\begin{array}{cc}
Z^{T} A Z & Z^{T} D \\
D^{T} Z & -C
\end{array}\right]\left[\begin{array}{c}
\mathbf{p} \\
\delta \mathbf{U}
\end{array}\right]=-\left[\begin{array}{c}
Z^{T} \nabla_{\mathbf{n}} f \\
\nabla_{\mathbf{U}} f
\end{array}\right]
$$

6. update and normalize:

$$
\boldsymbol{\delta} \mathbf{n}=Z \mathbf{p}, \quad \mathbf{n}_{j}^{\mathrm{RN}}=\frac{\mathbf{n}_{j}+\boldsymbol{\delta} \mathbf{n}_{j}}{\left|\mathbf{n}_{j}+\boldsymbol{\delta} \mathbf{n}_{j}\right|}, \quad j=1, \ldots, n, \quad \mathbf{U}^{\mathrm{RN}}=\mathbf{U}+\boldsymbol{\delta} \mathbf{U}
$$

7. out: $\mathbf{n}^{\mathrm{RN}}, \mathbf{U}^{\mathrm{RN}}$ satisfying $\left|\mathbf{n}_{j}^{\mathrm{RN}}\right|=1, j=1, \ldots, n$

We note that the gradient $\nabla_{\mathbf{n}} f(\mathbf{n}, \mathbf{U})$ used to calculate $\boldsymbol{\lambda}$ above is already needed and that the calculation is simply done componentwise: $\lambda_{j}=-\nabla_{\mathbf{n}_{j}} f \cdot \mathbf{n}_{j}, j=1, \ldots, n$. The other matrix and vector components above are computed exactly as before, the Lagrange multipliers entering only in $A$. If we compare this modified step with the basic Newton step $\left(\mathbf{n}^{\mathrm{N}}, \boldsymbol{\lambda}^{\mathrm{N}}, \mathbf{U}^{\mathrm{N}}\right)$ from $(\mathbf{n}, \boldsymbol{\lambda}, \mathbf{U})$, with the same input $\mathbf{n}$ and $\mathbf{U}$ and with $\boldsymbol{\lambda}$ computed as above, we see that $\mathbf{n}^{\mathrm{RN}}$ is simply a renormalized version of $\mathbf{n}^{\mathrm{N}}=\mathbf{n}+\boldsymbol{\delta} \mathbf{n}$, $\boldsymbol{\lambda}$ is treated as an intermediary (and computed differently than $\boldsymbol{\lambda}^{\mathrm{N}}=\boldsymbol{\lambda}+\boldsymbol{\delta} \boldsymbol{\lambda}$, only when needed at the next step), and $\mathbf{U}^{\mathrm{RN}}=\mathbf{U}^{\mathrm{N}}=\mathbf{U}+\boldsymbol{\delta} \mathbf{U}$.

As is proven below, this scheme is locally quadratically convergent, and so stopping criteria generally used for Newton-like iterations can be used here. These can be based on relative nonlinear residuals of $Z^{T} \nabla_{\mathbf{n}} f$ and $\nabla_{\mathbf{U}} f$, for example. More simply, one can just monitor the magnitudes of the corrections $\boldsymbol{\delta} \mathbf{n}$ and $\boldsymbol{\delta} \mathbf{U}$, relying on the property

$$
\left\|\mathbf{x}^{(k)}-\mathbf{x}^{*}\right\|=\left\|\mathbf{x}^{(k+1)}-\mathbf{x}^{(k)}\right\|+O\left(\left\|\mathbf{x}^{(k)}-\mathbf{x}^{*}\right\|^{2}\right), \quad \mathbf{x}=(\mathbf{n}, \mathbf{U}), \quad \mathbf{x}^{*}=\left(\mathbf{n}^{*}, \mathbf{U}^{*}\right) .
$$


The entries of $\mathbf{n}$ are $O(1)$ by nature. If the problem has been well scaled (as in $\S \mathrm{S} 1.3$ ), then so will the entries of $\mathbf{U}$ be. Otherwise, one can take into account a scale factor for $\mathbf{U}$ and use a stopping condition of the form

$$
\|\boldsymbol{\delta} \mathbf{n}\|_{\infty} \leq \text { tol and }\|\boldsymbol{\delta} \mathbf{U}\|_{\infty} \leq \text { tol } *(\text { scale for } U) .
$$

See for example $[15, \S 5.2]$.

Besides the modest analytical simplifications gained by this scheme, it is physically intuitive and somewhat analogous to other numerical approaches that have been applied to related problems. The numerical device of renormalizing after each step has been used by computational physicists in this area for a long time (in the context of the pseudo-time-relaxation approach to computing constrained equilibria) and has also been employed by numerical analysts in the context of both relaxation and gradient methods [1, 3, 17]. Analogous ideas have been used in the area of micromagnetics - see [8, $\S 4],[16, \S 2.2 .1]$, or [20, Ch. 4]. In $\S 4.3$ below, we explore the relationship of the Renormalized Newton Method to the Truncated Newton Method of computational micromagnetics.

4.1. Local quadratic convergence analysis of the Renormalized Newton Method. In spite of the attractive features discussed above, one would not contemplate using such an alternative outer iteration if it did not preserve the quadratic local convergence properties of the basic Newton method. In fact it does, which we now prove. The analysis relies upon two facts: first, that the error in $\boldsymbol{\lambda}$ computed by (4.1) as an approximation to the Lagrange multiplier vector $\boldsymbol{\lambda}^{*}$ of the exact solution $\left(\mathbf{n}^{*}\right.$, $\mathbf{U}^{*}$ ) is of the same order as the errors in $\mathbf{n}-\mathbf{n}^{*}$ and $\mathbf{U}-\mathbf{U}^{*}$, and second, that the renormalization step is second order in the Newton correction $\boldsymbol{\delta} \mathbf{n}$. We establish these preliminary results in two lemmas, after first introducing some notation and recalling some local convergence results for the basic global Newton iteration.

Let $\mathbf{x}^{*}=\left(\mathbf{n}^{*}, \boldsymbol{\lambda}^{*}, \mathbf{U}^{*}\right)$ be a regular discrete constrained equilibrium solution, that is,

$$
\nabla L\left(\mathbf{x}^{*}\right)=\mathbf{0}, \quad \nabla^{2} L\left(\mathbf{x}^{*}\right) \text { non-singular. }
$$

We note that this can include locally unstable solutions as well as locally stable solutions. The only situation excluded is that of singular equilibrium solutions, at which $\nabla L\left(\mathbf{x}^{*}\right)=\mathbf{0}$ but the Hessian $\nabla^{2} L\left(\mathbf{x}^{*}\right)$ is singular - in a typical parameter study, such situations would occur at bifurcation and turning points, for example.

For convenience, we work with vector and matrix 2-norms, and adopt the notation

$$
\begin{aligned}
\mathcal{B}_{\varepsilon}\left(\mathbf{x}^{*}\right) & =\left\{\mathbf{x} \mid\left\|\mathbf{x}-\mathbf{x}^{*}\right\|_{2} \leq \varepsilon\right\} \\
& =\left\{(\mathbf{n}, \boldsymbol{\lambda}, \mathbf{U}) \mid\left\|\mathbf{n}-\mathbf{n}^{*}\right\|_{2}^{2}+\left\|\boldsymbol{\lambda}-\boldsymbol{\lambda}^{*}\right\|_{2}^{2}+\left\|\mathbf{U}-\mathbf{U}^{*}\right\|_{2}^{2} \leq \varepsilon^{2}\right\}, \\
\mathcal{B}_{\varepsilon}^{\prime}\left(\mathbf{x}^{*}\right) & =\left\{(\mathbf{n}, \mathbf{U}) \mid\left\|\mathbf{n}-\mathbf{n}^{*}\right\|_{2}^{2}+\left\|\mathbf{U}-\mathbf{U}^{*}\right\|_{2}^{2} \leq \varepsilon^{2}\right\}, \\
\mathcal{B}_{\varepsilon}^{\prime \prime}\left(\mathbf{x}^{*}\right) & =\left\{(\mathbf{n}, \mathbf{U}) \in \mathcal{B}_{\varepsilon}^{\prime}\left(\mathbf{x}^{*}\right)|| \mathbf{n}_{j} \mid=1, j=1, \ldots, n\right\} .
\end{aligned}
$$

We recall that we assume that our problem has been non-dimensionalized, so that $\mathbf{n}$, $\boldsymbol{\lambda}, \mathbf{U}$, and $\mathbf{x}$ are all dimensionless. We note that $\mathcal{B}_{\varepsilon}^{\prime}\left(\mathbf{x}^{*}\right)$ corresponds to the $\boldsymbol{\lambda}=\boldsymbol{\lambda}^{*}$ section of $\mathcal{B}_{\varepsilon}\left(\mathbf{x}^{*}\right)$ and that $(\mathbf{n}, \boldsymbol{\lambda}, \mathbf{U}) \in \mathcal{B}_{\varepsilon}\left(\mathbf{x}^{*}\right)$ implies $(\mathbf{n}, \mathbf{U}) \in \mathcal{B}_{\varepsilon}^{\prime}\left(\mathbf{x}^{*}\right)$, while $\mathcal{B}_{\varepsilon}^{\prime \prime}\left(\mathbf{x}^{*}\right)$ is a subset of $\mathcal{B}_{\varepsilon}^{\prime}\left(\mathbf{x}^{*}\right)$, adding only the requirement that $\mathbf{n}$ be normalized. For conventional discretizations, the discrete Lagrangian $L$ is an algebraic function (a multivariate polynomial in the components of $\mathbf{x}$ ) and is therefore infinitely continuously differentiable. 
As such, it satisfies any needed regularity hypotheses, and standard results on the local convergence of Newton's Method hold - see for example [4, §5.2], [15, §5.1], or $[19, \S 10.2 .2]$. A sufficient (lesser) hypothesis for the analysis that follows is that the Hessian $\nabla^{2} L$ be Lipschitz continuous on a neighborhood of $\mathbf{x}^{*}$.

TheOrem 4.2 (Local Newton Convergence). Let $\mathbf{x}^{*}$ be a regular critical point of $L$, as in (4.2), with $\nabla^{2} L$ Lipschitz continuous on a neighborhood of $\mathbf{x}^{*}$, then there exist positive constants $C_{\mathrm{N}}$ and $\varepsilon_{\mathrm{N}}$ satisfying $C_{\mathrm{N}} \varepsilon_{\mathrm{N}}<1$, such that for any $\mathbf{x} \in \mathcal{B}_{\varepsilon_{\mathrm{N}}}\left(\mathbf{x}^{*}\right)$, the Newton step $\mathbf{x}^{\mathrm{N}}$ is well defined and satisfies

$$
\left\|\mathrm{x}^{\mathrm{N}}-\mathrm{x}^{*}\right\|_{2} \leq C_{\mathrm{N}}\left\|\mathrm{x}-\mathrm{x}^{*}\right\|_{2}^{2}
$$

We recall that from this basic estimate it follows that the Newton iteration is quadratically convergent from any initial guess $\mathbf{x}^{(0)} \in \mathcal{B}_{\varepsilon_{\mathrm{N}}}\left(\mathbf{x}^{*}\right)$ by arguing as follows:

$$
\left\|\mathbf{x}^{(1)}-\mathbf{x}^{*}\right\|_{2} \leq C_{\mathrm{N}}\left\|\mathbf{x}^{(0)}-\mathbf{x}^{*}\right\|_{2}^{2} \leq C_{\mathrm{N}} \varepsilon_{\mathrm{N}}\left\|\mathbf{x}^{(0)}-\mathbf{x}^{*}\right\|_{2},
$$

which implies $\left\{\mathbf{x}^{(k)}\right\}_{k=0}^{\infty} \subset \mathcal{B}_{\varepsilon_{\mathrm{N}}}\left(\mathbf{x}^{*}\right)\left(\right.$ since $\left.C_{\mathrm{N}} \varepsilon_{\mathrm{N}}<1\right)$,

$$
\left\|\mathbf{x}^{(k)}-\mathbf{x}^{*}\right\|_{2} \leq\left(C_{\mathrm{N}} \varepsilon_{\mathrm{N}}\right)^{k}\left\|\mathbf{x}^{(0)}-\mathbf{x}^{*}\right\|_{2} \rightarrow 0, \text { as } k \rightarrow \infty,
$$

and

$$
\left\|\mathbf{x}^{(k+1)}-\mathbf{x}^{*}\right\|_{2} \leq C_{\mathrm{N}}\left\|\mathbf{x}^{(k)}-\mathbf{x}^{*}\right\|_{2}^{2}, \quad k=0,1, \ldots .
$$

Our objective here is to establish an analogous result for the Renormalized Newton scheme. The following lemma shows that if $(\mathbf{n}, \mathbf{U})$ is sufficiently close to $\left(\mathbf{n}^{*}, \mathbf{U}^{*}\right)$, then (n, $\boldsymbol{\lambda}, \mathbf{U})$ (with $\boldsymbol{\lambda}$ calculated using (4.1)) is guaranteed to be within the region of attraction of the basic Newton iteration.

Lemma 4.3. Let $\mathbf{x}^{*}$ be a regular critical point of $L$, as in (4.2), with $\nabla^{2} L$ Lipschitz continuous on a neighborhood of $\mathbf{x}^{*}$, then there exist positive constants $C_{1}$ and $\varepsilon_{1}$ such that

$$
(\mathbf{n}, \mathbf{U}) \in \mathcal{B}_{\varepsilon_{\mathrm{N}}}^{\prime}\left(\mathbf{x}^{*}\right) \Rightarrow\left\|\boldsymbol{\lambda}-\boldsymbol{\lambda}^{*}\right\|_{2} \leq C_{1} \sqrt{\left\|\mathbf{n}-\mathbf{n}^{*}\right\|_{2}^{2}+\left\|\mathbf{U}-\mathbf{U}^{*}\right\|_{2}^{2}}
$$

and

$$
(\mathbf{n}, \mathbf{U}) \in \mathcal{B}_{\varepsilon_{1}}^{\prime}\left(\mathbf{x}^{*}\right) \Rightarrow(\mathbf{n}, \boldsymbol{\lambda}, \mathbf{U}) \in \mathcal{B}_{\varepsilon_{\mathrm{N}}}\left(\mathbf{x}^{*}\right),
$$

with $\boldsymbol{\lambda}$ computed from $(\mathbf{n}, \mathbf{U})$ using (4.1). Here $\varepsilon_{\mathrm{N}}$ is the local Newton radius of Theorem 4.2.

Proof. Let $(\mathbf{n}, \mathbf{U})$ be in $\mathcal{B}_{\varepsilon_{\mathrm{N}}}^{\prime}\left(\mathbf{x}^{*}\right)$. The approximate $\boldsymbol{\lambda}$ computed using (4.1) and the exact Lagrange multiplier vector $\boldsymbol{\lambda}^{*}$ satisfy

$$
\boldsymbol{\lambda}=-B(\mathbf{n})^{T} \nabla_{\mathbf{n}} f(\mathbf{n}, \mathbf{U}), \quad \boldsymbol{\lambda}^{*}=-B\left(\mathbf{n}^{*}\right)^{T} \nabla_{\mathbf{n}} f\left(\mathbf{n}^{*}, \mathbf{U}^{*}\right) .
$$

Subtracting these and using the fact that the matrix function $B$ is linear in its argument $\left(B(\mathbf{n})=B\left(\mathbf{n}-\mathbf{n}^{*}\right)+B\left(\mathbf{n}^{*}\right)\right)$, we obtain

$$
\boldsymbol{\lambda}^{*}-\boldsymbol{\lambda}=B\left(\mathbf{n}^{*}\right)^{T}\left[\nabla_{\mathbf{n}} f(\mathbf{n}, \mathbf{U})-\nabla_{\mathbf{n}} f\left(\mathbf{n}^{*}, \mathbf{U}^{*}\right)\right]+B\left(\mathbf{n}-\mathbf{n}^{*}\right)^{T} \nabla_{\mathbf{n}} f(\mathbf{n}, \mathbf{U}),
$$

which implies

$$
\begin{array}{rl}
\left\|\boldsymbol{\lambda}-\boldsymbol{\lambda}^{*}\right\|_{2} \leq\left\|B\left(\mathbf{n}^{*}\right)^{T}\right\|_{2} \| \nabla_{\mathbf{n}} f(\mathbf{n}, \mathbf{U})-\nabla_{\mathbf{n}} & f\left(\mathbf{n}^{*}, \mathbf{U}^{*}\right) \|_{2} \\
& +\left\|B\left(\mathbf{n}-\mathbf{n}^{*}\right)^{T}\right\|_{2}\left\|\nabla_{\mathbf{n}} f(\mathbf{n}, \mathbf{U})\right\|_{2} .
\end{array}
$$


The difference of the gradients above can be estimated using remainder formulas, such as in $[4, \S 4.1]$ or $[19, \S 3.2]$ :

$$
\begin{aligned}
\| \nabla_{\mathbf{n}} f(\mathbf{n}, \mathbf{U})- & \nabla_{\mathbf{n}} f\left(\mathbf{n}^{*}, \mathbf{U}^{*}\right) \|_{2} \\
\leq & \max _{0 \leq t \leq 1}\left\|\nabla_{\mathbf{n n}}^{2} f\left(\mathbf{n}^{*}+t\left(\mathbf{n}-\mathbf{n}^{*}\right), \mathbf{U}^{*}+t\left(\mathbf{U}-\mathbf{U}^{*}\right)\right)\right\|_{2}\left\|\mathbf{n}-\mathbf{n}^{*}\right\|_{2} \\
& \quad+\max _{0 \leq t \leq 1}\left\|\nabla_{\mathbf{n U}}^{2} f\left(\mathbf{n}^{*}+t\left(\mathbf{n}-\mathbf{n}^{*}\right), \mathbf{U}^{*}+t\left(\mathbf{U}-\mathbf{U}^{*}\right)\right)\right\|_{2}\left\|\mathbf{U}-\mathbf{U}^{*}\right\|_{2} \\
\leq & M_{1}\left\|\mathbf{n}-\mathbf{n}^{*}\right\|_{2}+M_{2}\left\|\mathbf{U}-\mathbf{U}^{*}\right\|_{2},
\end{aligned}
$$

with

$$
M_{1}:=\max _{(\mathbf{n}, \mathbf{U}) \in \mathcal{B}_{\varepsilon_{\mathrm{N}}}^{\prime}\left(\mathbf{x}^{*}\right)}\left\|\nabla_{\mathbf{n n}}^{2} f(\mathbf{n}, \mathbf{U})\right\|_{2}, \quad M_{2}:=\max _{(\mathbf{n}, \mathbf{U}) \in \mathcal{B}_{\varepsilon_{\mathbf{N}}}^{\prime}\left(\mathbf{x}^{*}\right)}\left\|\nabla_{\mathbf{n} \mathbf{U}}^{2} f(\mathbf{n}, \mathbf{U})\right\|_{2} .
$$

The matrix 2-norms of $B\left(\mathbf{n}^{*}\right)^{T}$ and $B\left(\mathbf{n}-\mathbf{n}^{*}\right)^{T}$ can be estimated by observing that they both stem from matrices of the general form

$$
B(\mathbf{b})=\left[\begin{array}{lll}
\mathbf{b}_{1} & & \\
& \ddots & \\
& & \mathbf{b}_{n}
\end{array}\right], \quad \mathbf{b}=\left(\mathbf{b}_{1}, \ldots, \mathbf{b}_{n}\right), \quad \mathbf{b}_{1}, \ldots, \mathbf{b}_{n} \in \mathbb{R}^{3},
$$

for which

$$
B(\mathbf{b}) B(\mathbf{b})^{T}=\left[\begin{array}{lll}
\mathbf{b}_{1} \mathbf{b}_{1}^{T} & & \\
& \ddots & \\
& & \mathbf{b}_{n} \mathbf{b}_{n}^{T}
\end{array}\right]
$$

It follows that

$$
\left\|B(\mathbf{b})^{T}\right\|_{2}=\sqrt{\lambda_{\max }\left(B(\mathbf{b}) B(\mathbf{b})^{T}\right)}=\max \left\{\left|\mathbf{b}_{1}\right|, \ldots,\left|\mathbf{b}_{n}\right|\right\} .
$$

Thus

$$
\left\|B\left(\mathbf{n}^{*}\right)^{T}\right\|_{2}=\max \left\{\left|\mathbf{n}_{1}^{*}\right|, \ldots,\left|\mathbf{n}_{n}^{*}\right|\right\}=1
$$

and

$$
\left\|B\left(\mathbf{n}-\mathbf{n}^{*}\right)^{T}\right\|_{2}=\max \left\{\left|\mathbf{n}_{1}-\mathbf{n}_{1}^{*}\right|, \ldots,\left|\mathbf{n}_{n}-\mathbf{n}_{n}^{*}\right|\right\} \leq\left\|\mathbf{n}-\mathbf{n}^{*}\right\|_{2} .
$$

With the help of these estimates, it follows from (4.4) that

$$
\left\|\boldsymbol{\lambda}-\boldsymbol{\lambda}^{*}\right\|_{2} \leq M_{1}\left\|\mathbf{n}-\mathbf{n}^{*}\right\|_{2}+M_{2}\left\|\mathbf{U}-\mathbf{U}^{*}\right\|_{2}+M_{3}\left\|\mathbf{n}-\mathbf{n}^{*}\right\|_{2},
$$

with

$$
M_{3}:=\max _{(\mathbf{n}, \mathbf{U}) \in \mathcal{B}_{\varepsilon_{\mathrm{N}}}^{\prime}\left(\mathbf{x}^{*}\right)}\left\|\nabla_{\mathbf{n}} f(\mathbf{n}, \mathbf{U})\right\|_{2},
$$

which in turn implies that

$$
\left\|\boldsymbol{\lambda}-\boldsymbol{\lambda}^{*}\right\|_{2} \leq C_{1} \sqrt{\left\|\mathbf{n}-\mathbf{n}^{*}\right\|_{2}^{2}+\left\|\mathbf{U}-\mathbf{U}^{*}\right\|_{2}^{2}} \text {, with } C_{1}:=\sqrt{\left(M_{1}+M_{3}\right)^{2}+M_{2}^{2}} .
$$


If we now take

$$
\varepsilon_{1}:=\frac{\varepsilon_{\mathrm{N}}}{\sqrt{C_{1}^{2}+1}}
$$

we obtain

$$
\begin{aligned}
& (\mathbf{n}, \mathbf{U}) \in \mathcal{B}_{\varepsilon_{1}}^{\prime}\left(\mathbf{x}^{*}\right) \Rightarrow \\
& \left\|\mathbf{n}-\mathbf{n}^{*}\right\|_{2}^{2}+\left\|\boldsymbol{\lambda}-\boldsymbol{\lambda}^{*}\right\|_{2}^{2}+\left\|\mathbf{U}-\mathbf{U}^{*}\right\|_{2}^{2} \leq\left(C_{1}^{2}+1\right)\left(\left\|\mathbf{n}-\mathbf{n}^{*}\right\|_{2}^{2}+\left\|\mathbf{U}-\mathbf{U}^{*}\right\|_{2}^{2}\right) \\
& \leq\left(C_{1}^{2}+1\right) \varepsilon_{1}^{2}=\varepsilon_{\mathrm{N}}^{2} \Rightarrow(\mathbf{n}, \boldsymbol{\lambda}, \mathbf{U}) \in \mathcal{B}_{\varepsilon_{\mathrm{N}}}\left(\mathbf{x}^{*}\right) .
\end{aligned}
$$

The above is consistent with general results for "least squares multipliers"- see for example $[11, \S 6.6]$ or $[18, \S 18.3]$. Thus, for $(\mathbf{n}, \mathbf{U}) \in \mathcal{B}_{\varepsilon_{1}}^{\prime}\left(\mathbf{x}^{*}\right)$, the Newton step (from ( $\mathbf{n}, \boldsymbol{\lambda}, \mathbf{U}$ ), with $\boldsymbol{\lambda}$ as in (4.1)) is well defined, and hence so is the Renormalized Newton step. Our next preliminary result shows that the renormalization step is second order in $\boldsymbol{\delta} \mathbf{n}$ and therefore preserves the order of accuracy of the basic Newton step.

Lemma 4.4. Let $\mathbf{x}^{*}$ be a regular critical point of L, as in (4.2), with $\nabla^{2} L$ Lipschitz continuous on a neighborhood of $\mathbf{x}^{*}$, then there exists a positive constant $C_{2}$ such that

$$
(\mathbf{n}, \mathbf{U}) \in \mathcal{B}_{\varepsilon_{1}}^{\prime \prime}\left(\mathbf{x}^{*}\right) \Rightarrow\left\|\mathbf{n}^{\mathrm{RN}}-\mathbf{n}^{*}\right\|_{2} \leq C_{2}\left\|\mathbf{x}-\mathbf{x}^{*}\right\|_{2}^{2},
$$

where $\varepsilon_{1}$ is as in Lemma 4.3 and $\mathbf{x}=(\mathbf{n}, \boldsymbol{\lambda}, \mathbf{U})$, with $\boldsymbol{\lambda}$ computed as in (4.1).

Proof. We know from Lemma 4.3 that for $(\mathbf{n}, \mathbf{U}) \in \mathcal{B}_{\varepsilon_{1}}^{\prime \prime}\left(\mathbf{x}^{*}\right) \subset \mathcal{B}_{\varepsilon_{1}}^{\prime}\left(\mathbf{x}^{*}\right)$, the point $(\mathbf{n}, \boldsymbol{\lambda}, \mathbf{U})$ is in $\mathcal{B}_{\varepsilon_{\mathrm{N}}}\left(\mathbf{x}^{*}\right)$ and that the Newton step from $(\mathbf{n}, \boldsymbol{\lambda}, \mathbf{U})$ is well defined and satisfies the inequality (4.3). The Renormalized Newton step is also well defined, and the local geometry relating the two is as follows. For $j=1, \ldots, n$,

$$
\mathbf{n}_{j}^{\mathrm{N}}=\mathbf{n}_{j}+\boldsymbol{\delta} \mathbf{n}_{j}, \quad\left|\mathbf{n}_{j}\right|=1, \quad \mathbf{n}_{j} \cdot \boldsymbol{\delta} \mathbf{n}_{j}=0, \quad\left|\mathbf{n}_{j}^{\mathrm{N}}\right| \geq 1, \quad \mathbf{n}_{j}^{\mathrm{RN}}=\frac{\mathbf{n}_{j}^{\mathrm{N}}}{\left|\mathbf{n}_{j}^{\mathrm{N}}\right|},
$$

from which follows

$$
\mathbf{n}_{j}^{\mathrm{N}}-\mathbf{n}_{j}^{\mathrm{RN}}=\left(\left|\mathbf{n}_{j}^{\mathrm{N}}\right|-1\right) \mathbf{n}_{j}^{\mathrm{RN}}
$$

and

$$
\left|\boldsymbol{\delta} \mathbf{n}_{j}\right|^{2}=\left|\mathbf{n}_{j}^{\mathrm{N}}\right|^{2}-1=\left(\left|\mathbf{n}_{j}^{\mathrm{N}}\right|-1\right)\left(\mid \mathbf{n}_{j}^{\mathrm{N}}+1\right) \Rightarrow\left|\mathbf{n}_{j}^{\mathrm{N}}\right|-1=\frac{\left|\boldsymbol{\delta} \mathbf{n}_{j}\right|^{2}}{\left|\mathbf{n}_{j}^{\mathrm{N}}\right|+1} \leq \frac{1}{2}\left|\boldsymbol{\delta} \mathbf{n}_{j}\right|^{2} .
$$

With this we can estimate

$$
\left\|\mathbf{n}^{\mathrm{RN}}-\mathbf{n}^{\mathrm{N}}\right\|_{2} \leq \sum_{j=1}^{n}\left\|\mathbf{n}_{j}^{\mathrm{RN}}-\mathbf{n}_{j}^{\mathrm{N}}\right\|_{2} \leq \frac{1}{2} \sum_{j=1}^{n}\left|\boldsymbol{\delta} \mathbf{n}_{j}\right|^{2}=\frac{1}{2}\|\boldsymbol{\delta} \mathbf{n}\|_{2}^{2} .
$$

To proceed, we must relate the Newton correction $\boldsymbol{\delta} \mathbf{n}$ to the errors in the initial vectors $(\mathbf{n}, \boldsymbol{\lambda}, \mathbf{U})$, which can be done as follows:

$$
\begin{aligned}
& \mathbf{n}^{\mathrm{N}}=\mathbf{n}+\boldsymbol{\delta} \mathbf{n} \Rightarrow \mathbf{n}^{\mathrm{N}}-\mathbf{n}^{*}=\mathbf{n}-\mathbf{n}^{*}+\boldsymbol{\delta} \mathbf{n} \\
& \Rightarrow\|\boldsymbol{\delta} \mathbf{n}\|_{2} \leq\left\|\mathbf{n}^{\mathrm{N}}-\mathbf{n}^{*}\right\|_{2}+\left\|\mathbf{n}-\mathbf{n}^{*}\right\|_{2} \\
& \leq C_{\mathrm{N}}\left\|\mathbf{x}-\mathbf{x}^{*}\right\|_{2}^{2}+\left\|\mathbf{x}-\mathbf{x}^{*}\right\|_{2}, \quad \text { using }(4.3) \\
& \leq\left(C_{\mathrm{N}} \varepsilon_{\mathrm{N}}+1\right)\left\|\mathbf{x}-\mathbf{x}^{*}\right\|_{2}, \quad \text { using }\left\|\mathbf{x}-\mathbf{x}^{*}\right\|_{2} \leq \varepsilon_{\mathrm{N}} \\
& \leq 2\left\|\mathbf{x}-\mathbf{x}^{*}\right\|_{2}, \quad \text { using } C_{\mathrm{N}} \varepsilon_{\mathrm{N}}<1 \\
& \Rightarrow \quad \frac{1}{2}\|\boldsymbol{\delta} \mathbf{n}\|_{2}^{2} \leq 2\left\|\mathbf{x}-\mathbf{x}^{*}\right\|_{2}^{2}
\end{aligned}
$$


Combining (4.8) and (4.9) with (4.3), we obtain

$$
\begin{aligned}
\left\|\mathbf{n}^{\mathrm{RN}}-\mathbf{n}^{*}\right\|_{2} & \leq\left\|\mathbf{n}^{\mathrm{RN}}-\mathbf{n}^{\mathrm{N}}\right\|_{2}+\left\|\mathbf{n}^{\mathrm{N}}-\mathbf{n}^{*}\right\|_{2} \\
& \leq \frac{1}{2}\|\boldsymbol{\delta} \mathbf{n}\|_{2}^{2}+\left\|\mathbf{n}^{\mathrm{N}}-\mathbf{n}^{*}\right\|_{2} \\
& \leq\left(2+C_{\mathrm{N}}\right)\left\|\mathbf{x}-\mathbf{x}^{*}\right\|_{2}^{2} .
\end{aligned}
$$

Thus the lemma is proved with $C_{2}:=2+C_{\mathrm{N}}$.

With the help of these lemmas, we can now prove the basic result that establishes the local quadratic convergence of the Renormalized Newton Method algorithm.

TheOREM 4.5. Let $\mathbf{x}^{*}$ be a regular critical point of L, as in (4.2), with $\nabla^{2} L$ Lipschitz continuous on a neighborhood of $\mathbf{x}^{*}$, then there exist positive constants $C_{\mathrm{RN}}$ and $\varepsilon_{\mathrm{RN}}$ satisfying $C_{\mathrm{RN}} \varepsilon_{\mathrm{RN}}<1$, such that for any $(\mathbf{n}, \mathbf{U}) \in \mathcal{B}_{\varepsilon_{\mathrm{RN}}^{\prime \prime}}^{\prime \prime}\left(\mathbf{x}^{*}\right)$, the Renormalized Newton step $\left(\mathbf{n}^{\mathrm{RN}}, \mathbf{U}^{\mathrm{RN}}\right)$, calculated via Algorithm 4.1, is well defined and satisfies

$$
\sqrt{\left\|\mathbf{n}^{\mathrm{RN}}-\mathbf{n}^{*}\right\|_{2}^{2}+\left\|\mathbf{U}^{\mathrm{RN}}-\mathbf{U}^{*}\right\|_{2}^{2}} \leq C_{\mathrm{RN}}\left(\left\|\mathbf{n}-\mathbf{n}^{*}\right\|_{2}^{2}+\left\|\mathbf{U}-\mathbf{U}^{*}\right\|_{2}^{2}\right) .
$$

Proof. Given any $(\mathbf{n}, \mathbf{U})$ in $\mathcal{B}_{\varepsilon_{1}}^{\prime \prime}\left(\mathbf{x}^{*}\right)$, with $\varepsilon_{1}$ as in Lemma 4.3 , define $\boldsymbol{\lambda}$ as in (4.1). Lemma 4.3 guarantees that $\mathbf{x}=(\mathbf{n}, \boldsymbol{\lambda}, \mathbf{U}) \in \mathcal{B}_{\varepsilon_{\mathrm{N}}}\left(\mathbf{x}^{*}\right)$, and so the Newton and Renormalized Newton steps are well defined. Lemma 4.4 guarantees that

$$
\left\|\mathbf{n}^{\mathrm{RN}}-\mathbf{n}^{*}\right\|_{2} \leq C_{2}\left\|\mathbf{x}-\mathbf{x}^{*}\right\|_{2}^{2},
$$

with $C_{2}$ as in Lemma 4.4, and we also have

$$
\left\|\mathbf{U}^{\mathrm{RN}}-\mathbf{U}^{*}\right\|_{2} \leq C_{\mathrm{N}}\left\|\mathbf{x}-\mathbf{x}^{*}\right\|_{2}^{2}
$$

from (4.3), because $\mathbf{U}^{\mathrm{RN}}=\mathbf{U}^{\mathrm{N}}$. It follows that

$$
\sqrt{\left\|\mathbf{n}^{\mathrm{RN}}-\mathbf{n}^{*}\right\|_{2}^{2}+\left\|\mathbf{U}^{\mathrm{RN}}-\mathbf{U}^{*}\right\|_{2}^{2}} \leq \sqrt{C_{2}^{2}+C_{\mathrm{N}}^{2}}\left\|\mathbf{x}-\mathbf{x}^{*}\right\|_{2}^{2} .
$$

Now

$$
\begin{aligned}
\left\|\mathbf{x}-\mathbf{x}^{*}\right\|_{2}^{2} & =\left\|\mathbf{n}-\mathbf{n}^{*}\right\|_{2}^{2}+\left\|\boldsymbol{\lambda}-\boldsymbol{\lambda}^{*}\right\|_{2}^{2}+\left\|\mathbf{U}-\mathbf{U}^{*}\right\|_{2}^{2} \\
& \leq\left(C_{1}^{2}+1\right)\left(\left\|\mathbf{n}-\mathbf{n}^{*}\right\|_{2}^{2}+\left\|\mathbf{U}-\mathbf{U}^{*}\right\|_{2}^{2}\right),
\end{aligned}
$$

where $C_{1}$ is as in (4.7). We thus have

$$
\sqrt{\left\|\mathbf{n}^{\mathrm{RN}}-\mathbf{n}^{*}\right\|_{2}^{2}+\left\|\mathbf{U}^{\mathrm{RN}}-\mathbf{U}^{*}\right\|_{2}^{2}} \leq \sqrt{C_{2}^{2}+C_{\mathrm{N}}^{2}}\left(C_{1}^{2}+1\right)\left(\left\|\mathbf{n}-\mathbf{n}^{*}\right\|_{2}^{2}+\left\|\mathbf{U}-\mathbf{U}^{*}\right\|_{2}^{2}\right)
$$

and can take

$$
C_{\mathrm{RN}}:=\sqrt{C_{2}^{2}+C_{\mathrm{N}}^{2}}\left(C_{1}^{2}+1\right)
$$

and choose $\varepsilon_{\mathrm{RN}} \leq \varepsilon_{1}$ such that $C_{\mathrm{RN}} \varepsilon_{\mathrm{RN}}<1$.

One can now use exactly the same arguments as in the basic local Newton Convergence Theorem (sketched after the statement of Theorem 4.2) to show that the Renormalized Newton iteration is well defined and quadratically convergent from any initial point in $\mathcal{B}_{\varepsilon_{\mathrm{RN}}}^{\prime \prime}\left(\mathbf{x}^{*}\right)$. 
In the analysis above, the various constants $\left(C_{1}, C_{2}, \varepsilon_{1}, \ldots\right)$ depend on the discrete Lagrangian $L$ in (3.1) through the bounds $M_{1}, M_{2}$, and $M_{3}$ in (4.5) and (4.6), and these will change from problem to problem. For example, consider the model problem in two dimensions used for the numerical experiments in $\S 5$ :

$$
\mathcal{F}[\boldsymbol{n}]=\frac{1}{2} \int_{\Omega}|\nabla \boldsymbol{n}|^{2}, \quad \boldsymbol{n}=\boldsymbol{n}(x, y), \quad \Omega=(0,1)^{2} .
$$

Discretized as in $\S 5$ on a uniform mesh with $n^{2}$ mesh cells of edge length $h=1 / n$ (and $(n-1)^{2}$ free interior nodes), one has the discrete free energy

$$
\begin{array}{r}
f(\mathbf{n})=\frac{1}{4} \sum_{i, j=0}^{n-1}\left[\left|\mathbf{n}_{i+1, j}-\mathbf{n}_{i j}\right|^{2}+\left|\mathbf{n}_{i+1, j+1}-\mathbf{n}_{i, j+1}\right|^{2}\right. \\
\left.+\left|\mathbf{n}_{i, j+1}-\mathbf{n}_{i j}\right|^{2}+\left|\mathbf{n}_{i+1, j+1}-\mathbf{n}_{i+1, j}\right|^{2}\right] .
\end{array}
$$

Formulas for $\nabla_{\mathbf{n}} f$ and $\nabla_{\mathbf{n n}}^{2} f$ are given in (5.2) and (5.5) respectively. Note that for this model problem, $A_{0}=\nabla_{\mathbf{n n}}^{2} f$ is constant, independent of $\mathbf{n}$, and $\nabla_{\mathbf{n}} f(\mathbf{n})=A_{0} \mathbf{n}$ (see $\S 5.1)$.

The constants $M_{1}$ and $M_{3}$ can be estimated from these formulas-there is no electric field in this model problem, and so there is no $M_{2}$ to estimate. For $M_{1}$ we obtain an exact expression:

$$
\begin{aligned}
M_{1}=\max _{\mathbf{n} \in \mathcal{B}_{\varepsilon_{\mathrm{N}}}\left(\mathbf{n}^{*}\right)}\left\|\nabla_{\mathbf{n n}}^{2} f(\mathbf{n})\right\|_{2}=\left\|A_{0}\right\|_{2}=\lambda_{\max }\left(A_{0}\right)=8 \sin ^{2} \frac{\pi(n-1)}{2 n} \\
\Rightarrow \quad M_{1}<8 \text { and } M_{1}=8\left(1+O\left(h^{2}\right)\right), \text { as } h \rightarrow 0 .
\end{aligned}
$$

For $M_{3}$ we obtain a bound:

$$
\begin{aligned}
\left\|\nabla_{\mathbf{n}} f(\mathbf{n})\right\|_{2}=\left\|A_{0} \mathbf{n}\right\|_{2} \leq\left\|A_{0}\right\|_{2}\left(\left\|\mathbf{n}-\mathbf{n}^{*}\right\|_{2}+\left\|\mathbf{n}^{*}\right\|_{2}\right) & \\
& \Rightarrow M_{3}=\max _{\mathbf{n} \in \mathcal{B}_{\varepsilon_{\mathrm{N}}}\left(\mathbf{n}^{*}\right)}\left\|\nabla_{\mathbf{n}} f(\mathbf{n})\right\|_{2} \leq 8\left(\varepsilon_{\mathrm{N}}+n\right) .
\end{aligned}
$$

Here we have used (4.10), the definition of $\mathcal{B}_{\mathcal{E}_{\mathrm{N}}}\left(\mathbf{n}^{*}\right)$, and $\left\|\mathbf{n}^{*}\right\|_{2}^{2}=\sum_{i, j=1}^{n-1}\left|\mathbf{n}_{i j}^{*}\right|^{2}=$ $(n-1)^{2}$ (since $\left.\left|\mathbf{n}_{i j}^{*}\right|=1, \forall i, j\right)$. That $\left\|\mathbf{n}^{*}\right\|_{2}=n-1$ (and $\| \nabla_{\mathbf{n}} f\left(\mathbf{n}^{*}\right)=O(n)$ above) is due to the definition of the vector 2-norm (the standard definition, as used above). Thus for this example we have $M_{1}=O(1)$ and $M_{3}=O(n)$, as $n \rightarrow \infty$. If the vector 2-norm were defined instead to be consistent with the continuous 2-norm (e.g., $\left.\|\mathbf{n}\|_{2}^{2}=h^{2} \sum_{i, j=1}^{n-1}\left|\mathbf{n}_{i j}\right|^{2}\right)$, then both $\left\|\mathbf{n}^{*}\right\|_{2}$ and $\left\|\nabla_{\mathbf{n}} f(\mathbf{n})\right\|_{2}$ would be $O(1)$, and $M_{1}$ would remain $O(1)$.

If the same model problem were discretized in the same way in one or three space dimensions (with $\Omega=(0,1)$ or $\Omega=(0,1)^{3}$ respectively, say), then we would obtain instead $M_{1}=O(1 / h), M_{3}=O\left(1 / h^{2}\right)$ (in one dimension) and $M_{1}=O(h), M_{3}=O(1)$ (in three dimensions). The analysis in this sub-section is for a problem (in any space dimension) on a fixed grid. Those familiar with the analysis of the "harmonic mapping case" in [1] will wonder if any of those results are relevant here. That analysis relies upon a certain "energy decay property" of the Dirichlet energy under renormalizing an approximate director field of greater than unit length. Unfortunately that property does not hold for general liquid crystal free-energy functionals, which we show in $\S \mathrm{S} 2$ of the Supplementary Material. 
4.2. Director fields with defects. An issue that could complicate matters is the possible presence of defects in the domain of the liquid crystal. These are singularities in the director field $\boldsymbol{n}$, usually caused by boundary conditions or some other form of frustration. The two canonical examples are radial line and point sources:

$$
\boldsymbol{n}(x, y)=\frac{1}{r}\left[\left(x-x_{\mathrm{d}}\right) \boldsymbol{e}_{x}+\left(y-y_{\mathrm{d}}\right) \boldsymbol{e}_{y}\right], \quad r^{2}=\left(x-x_{\mathrm{d}}\right)^{2}+\left(y-y_{\mathrm{d}}\right)^{2}
$$

and

$$
\begin{aligned}
\boldsymbol{n}(x, y, z) & =\frac{1}{r}\left[\left(x-x_{\mathrm{d}}\right) \boldsymbol{e}_{x}+\left(y-y_{\mathrm{d}}\right) \boldsymbol{e}_{y}+\left(z-z_{\mathrm{d}}\right) \boldsymbol{e}_{z}\right] \\
r^{2} & =\left(x-x_{\mathrm{d}}\right)^{2}+\left(y-y_{\mathrm{d}}\right)^{2}+\left(z-z_{\mathrm{d}}\right)^{2} .
\end{aligned}
$$

The first is referred to as a "disclination line" (and is uniform in the $z$ direction), and the second, a "point defect." The director field is undefined at the defect location, and the singularity is not removable. For the fields above, one can calculate

$$
|\nabla \boldsymbol{n}|=\left\{\begin{array}{l}
\frac{1}{r}, \text { for }(4.12) \\
\frac{2}{r}, \text { for }(4.13)
\end{array} .\right.
$$

We see that $|\nabla \boldsymbol{n}|^{2}$ is integrable in 3-D but not in 2-D, and so point defects have finite free energy in Oseen-Frank models, while disclination lines do not.

The analysis of [13] shows that the equilibrium director fields associated with Oseen-Frank models (with or without electric and/or magnetic fields) are infinitely differentiable in the interior of the domain away from defects. In many liquid-crystalbased devices or experiments, defects are not present; in many other cases, however, they are. The accurate numerical modeling of director fields with defects is a challenging problem in its own right and is beyond the scope of this paper. Local mesh refinement and such are required to resolve the rapid variation of $\boldsymbol{n}$ near the defect, and some cutoff or excision is required, since the director field is not even defined at the defect location. If one were to model a director field with a defect using a finite-difference grid or finite-element mesh, then the free-energy-minimizing discrete director field would position the defect location off the grid/mesh, and calculated solutions would sometimes obtain a slightly inaccurate defect location due to "trapping" of the defect in the wrong grid cell.

The analysis of the previous sub-section should remain valid for most reasonable discretizations (on a fixed grid or mesh) of problems with defects, although one would expect the various constants and bounds and regions of attraction of the Newton schemes to be affected. The analysis certainly remains valid for the case of standard finite-element schemes: for a given fixed mesh, the discrete Lagrangian is still just a polynomial function of the components of the discrete director $\mathbf{n}$, the Lagrange multipliers $\boldsymbol{\lambda}$, and the potential $\mathbf{U}$, and the bounds $M_{1}, M_{2}$, and $M_{3}$ are still finite. In numerical experiments in $\S 5$ below, we illustrate the robustness of both the basic Newton iteration and the Renormalized Newton Method for a model problem with a defect.

4.3. Comparison with the Truncated Newton Method of computational micromagnetics. The closest analogue to the Renormalized Newton scheme 
of which we are aware is the Truncated Newton Method utilized in micromagnetics $[8, \S 4.2]$, and it is natural and interesting to compare the two. The Truncated Newton Method (as adapted to micromagnetics) is used to minimize a discretization of the Landau-Lifshitz free energy of a ferromagnetic material subject to pointwise unit-length constraints on the normalized magnetization vector field, which is usually denoted by $\boldsymbol{m}$ and is analogous to the liquid crystal director field $\boldsymbol{n}$ - see the Supplementary Material. In our setting, the approach amounts to the following. Let $f\left(\mathbf{n}_{1}, \ldots, \mathbf{n}_{n}\right)$ be a discretized free energy, with $\mathbf{n}=\left(\mathbf{n}_{1}, \ldots, \mathbf{n}_{n}\right), \mathbf{n}_{j} \in \mathbb{R}^{3}$ a current approximate director (or magnetization) field, normalized so that $\left|\mathbf{n}_{j}\right|=1, \forall j$. One develops a constrained local quadratic model using paths of the form

$$
\mathbf{n}_{j}(\varepsilon)=\frac{\mathbf{n}_{j}+\varepsilon \mathbf{p}_{j}}{\left|\mathbf{n}_{j}+\varepsilon \mathbf{p}_{j}\right|}, \quad-\varepsilon_{0}<\varepsilon<\varepsilon_{0}, \quad \text { so that }\left|\mathbf{n}_{j}(\varepsilon)\right|=1 \text { and } \mathbf{n}_{j}(0)=\mathbf{n}_{j} .
$$

Here $\mathbf{p}$ is an arbitrary direction

$$
\mathbf{p}=\left[\begin{array}{c}
\mathbf{p}_{1} \\
\vdots \\
\mathbf{p}_{n}
\end{array}\right], \quad \mathbf{p}_{1}, \ldots, \mathbf{p}_{n} \in \mathbb{R}^{3}
$$

We note that this kind of device is commonly used for analytical as well as numerical purposes in both the liquid crystals and the micromagnetics areas - see for example $[23, \S 3.5]$, where it is used systematically to derive constrained equilibrium equations, natural boundary conditions, and the like for the Oseen-Frank elastic model for nematic liquid crystals. The constrained local quadratic model follows by expanding

$$
f\left(\mathbf{n}_{1}(\varepsilon), \ldots, \mathbf{n}_{n}(\varepsilon)\right)=f(\mathbf{n})+\varepsilon \mathbf{G}(\mathbf{n}) \cdot \mathbf{p}+\frac{\varepsilon^{2}}{2} H(\mathbf{n}) \mathbf{p} \cdot \mathbf{p}+\cdots,
$$

where $\mathbf{G}(\mathbf{n})$ and $H(\mathbf{n})$ are the constrained/projected gradient and Hessian evaluated at $\mathbf{n}(0)=\mathbf{n}$. The constrained Newton direction is characterized by

$$
H(\mathbf{n}) \mathbf{p}=-\mathbf{G}(\mathbf{n}),
$$

which corresponds to Eqn. (65) of [8] — here we have absorbed the $\varepsilon$ into $\mathbf{p}$.

If one performs the necessary calculus, one finds that

$$
\mathbf{G}=\left[\begin{array}{c}
\mathbf{G}_{1} \\
\vdots \\
\mathbf{G}_{n}
\end{array}\right], \quad \mathbf{G}_{j}=\nabla_{\mathbf{n}_{j}} f-\left(\nabla_{\mathbf{n}_{j}} f \cdot \mathbf{n}_{j}\right) \mathbf{n}_{j}=\Pi_{j} \nabla_{\mathbf{n}_{j}} f, \quad \Pi_{j}:=I-\mathbf{n}_{j} \mathbf{n}_{j}^{T},
$$

which is Eqn. (62) of [8]. Here $\Pi_{j}$ is the local orthogonal projector transverse to $\mathbf{n}_{j}$, which is commonly denoted $P(\boldsymbol{n})=\mathbf{I}-\boldsymbol{n} \otimes \boldsymbol{n}$ in the liquid crystals area (see [23]), and we can write

$$
\mathbf{G}=\Pi \nabla_{\mathbf{n}} f, \quad \Pi=\left[\begin{array}{lll}
\Pi_{1} & & \\
& \ddots & \\
& & \Pi_{n}
\end{array}\right] .
$$

Recall that in our Renormalized Newton Method, the approximate Lagrange multipliers are computed via (4.1) as

$$
\boldsymbol{\lambda}=-B(\mathbf{n})^{T} \nabla_{\mathbf{n}} f(\mathbf{n}) \Leftrightarrow \lambda_{j}=-\nabla_{\mathbf{n}_{j}} f \cdot \mathbf{n}_{j}, j=1, \ldots, n .
$$


In terms of this, then, we can write

$$
\mathbf{G}_{j}=\nabla_{\mathbf{n}_{j}} f+\lambda_{j} \mathbf{n}_{j} \Leftrightarrow \mathbf{G}=\nabla_{\mathbf{n}} f+B(\mathbf{n}) \boldsymbol{\lambda}=\nabla_{\mathbf{n}} L
$$

and we see that our formulas for the $\lambda_{j}$ arise naturally in this expansion calculus and that the projected gradient $\mathbf{G}$ is precisely the gradient with respect to $\mathbf{n}$ of the Lagrangian $L$ in (3.1) when the Lagrange multipliers are given by the formulas (4.17) above (which are the same as (4.1) before). The projected Hessian takes the form

$$
H=\Pi \nabla^{2} f \Pi+\Lambda \Pi-H_{2},
$$

where the diagonal matrix of approximate Lagrange multipliers $\Lambda$ is as given in (3.3) (again with $\lambda_{j}$ evaluated by (4.17) above), and

$$
H_{2}:=\left[\begin{array}{lll}
\mathbf{n}_{1} \mathbf{G}_{1}^{T}+\mathbf{G}_{1} \mathbf{n}_{1}^{T} & & \\
& \ddots & \\
& & \mathbf{n}_{n} \mathbf{G}_{n}^{T}+\mathbf{G}_{n} \mathbf{n}_{n}^{T}
\end{array}\right] .
$$

This is equivalent to Eqn. (63) of [8]. The $3 n \times 3 n$ matrices $H$ and $H_{2}$ are real and symmetric.

The projection $\Pi$ can be seen to be related to the matrices $B$ and $Z$ utilized in our Renormalized Newton algorithm via

$$
\Pi=I-B B^{T}=Z Z^{T} .
$$

Using also the observation that $\Lambda \Pi=\Pi \Lambda \Pi$, one is able to compare directly the Truncated Newton step

$$
H(\mathbf{n}) \mathbf{p}=-\mathbf{G}(\mathbf{n}), \quad H=Z Z^{T}\left(\nabla_{\mathbf{n n}}^{2} f+\Lambda\right) Z Z^{T}-H_{2}, \quad \mathbf{G}=Z Z^{T} \nabla_{\mathbf{n}} f
$$

with the Renormalized Newton step

$$
Z^{T}\left(\nabla_{\mathbf{n n}}^{2} f+\Lambda\right) Z \mathbf{q}=-Z^{T} \nabla_{\mathbf{n}} f, \quad \mathbf{p}=Z \mathbf{q} .
$$

In both instances, the Lagrange multipliers that form $\Lambda$ are given by the explicit formulas (4.1), (4.17). Observe that $\nabla_{\mathbf{n n}}^{2} f+\Lambda$ is simply the $A$ block of the Hessian of the Lagrangian $L$ in the notation of $\S 3$ here. Also note that at equilibrium, the projected gradient must necessarily vanish $\left(Z^{T} \nabla_{\mathbf{n}} f=\mathbf{0} \Rightarrow \mathbf{G}=Z Z^{T} \nabla_{\mathbf{n}} f=\mathbf{0}\right)$ and so, therefore, must the $H_{2}$ part of the projected Hessian matrix $H$. The convergence analysis of $\S 4.1$ here shows that this $H_{2}$ term is not needed for local quadratic convergence anyway.

We see that there is a definite relationship between the Truncated Newton Method of computational micromagnetics and the Renormalized Newton Method we have developed here. There are also important differences. The Truncated Newton system (4.19) is of size $3 n$, whereas (4.20) is of size $2 n$. This is reflective of the essential analytical difference between the two schemes: in the Truncated Newton step, the Hessian $\nabla_{\mathbf{n n}}^{2} L=\nabla_{\mathbf{n n}}^{2} f+\Lambda$ is flanked by a projection $\left(\Pi \mathbf{x}=Z Z^{T} \mathbf{x}\right.$ is the orthogonal projection of $\mathbf{x}$ onto the tangent space to the constraint manifold at $\mathbf{n}$ ), whereas in the Renormalized Newton step, the Hessian is flanked by a coordinate map $\left(Z^{T} \mathbf{x}\right.$ gives the coordinates of the tangent-space component of $\mathbf{x}$ with respect to the orthonormal basis provided by the columns of $Z$ ). The Truncated Newton scheme leaves the problem in $\mathbb{R}^{3 n}$ (even though there are only $2 n$ degrees of freedom locally on the constraint manifold), whereas the Renormalized Newton scheme moves the problem to a $2 n$ dimensional setting. The unnecessary extra degrees of freedom in the Truncated Newton approach contribute to the degeneracies identified in the next section. 
4.4. Convergence issues for the Truncated Newton Method. We further our comparison of the Renormalized Newton Method and the Truncated Newton Method, continuing to consider the problem of minimizing a general discretized free energy $f\left(\mathbf{n}_{1}, \ldots, \mathbf{n}_{n}\right)$ subject to $\left|\mathbf{n}_{j}\right|=1, j=1, \ldots, n$, with associated Lagrangian $L(\mathbf{n}, \boldsymbol{\lambda})=f(\mathbf{n})+\sum_{j=1}^{n} \lambda_{j} g_{j}(\mathbf{n})$, as in (3.1) (but with no electric field). At any regular constrained equilibrium point $\mathbf{n}^{*}$, we have $\nabla L\left(\mathbf{n}^{*}, \boldsymbol{\lambda}^{*}\right)=\mathbf{0}$ and $\nabla^{2} L\left(\mathbf{n}^{*}, \boldsymbol{\lambda}^{*}\right)$ nonsingular, where here

$$
\nabla^{2} L=\left[\begin{array}{cc}
\nabla_{\mathbf{n}}^{2} L & \nabla_{\mathbf{n} \boldsymbol{\lambda}}^{2} L \\
\nabla_{\boldsymbol{\lambda} \mathbf{n}}^{2} L & \nabla_{\boldsymbol{\lambda} \boldsymbol{\lambda}}^{2} L
\end{array}\right]=\left[\begin{array}{cc}
A & B \\
B^{T} & O
\end{array}\right], \quad A=\nabla_{\mathbf{n n}}^{2} f+\Lambda .
$$

With $\mathbf{n}^{*}$ normalized $\left(\left|\mathbf{n}_{j}^{*}\right|=1, \forall j\right)$ and $B$ and $Z$ constructed as before in (3.4) and (3.5) (mutually orthonormal columns, $B^{T} Z=O, Z^{T} B=O$ ), the Lagrange multipliers associated with $\mathbf{n}^{*}$ are given by $\boldsymbol{\lambda}^{*}=-B\left(\mathbf{n}^{*}\right)^{T} \nabla_{\mathbf{n}} f\left(\mathbf{n}^{*}\right)$, and it can be shown that

$$
\left[\begin{array}{cc}
A & B \\
B^{T} & O
\end{array}\right] \text { is nonsingular } \Leftrightarrow Z^{T} A Z \text { is nonsingular. }
$$

It follows that the coefficient matrix of the Renormalized Newton step (4.20), namely $Z^{T}\left(\nabla_{\mathbf{n n}}^{2} f+\Lambda\right) Z$, is nonsingular at $\left(\mathbf{n}^{*}, \boldsymbol{\lambda}^{*}\right)$, and by continuity at any $(\mathbf{n}, \boldsymbol{\lambda})$ sufficiently close to $\left(\mathbf{n}^{*}, \boldsymbol{\lambda}^{*}\right)$.

Consider on the other hand the coefficient matrix $H(\mathbf{n})$ (4.18) of the Truncated Newton step (4.19). As already observed, at $\mathbf{n}=\mathbf{n}^{*}$, we must have $\mathbf{G}\left(\mathbf{n}^{*}\right)=\mathbf{0}$, which implies that $H_{2}\left(\mathbf{n}^{*}\right)=O$ and

$$
H\left(\mathbf{n}^{*}\right)=\Pi\left(\nabla_{\mathbf{n n}}^{2} f+\Lambda\right) \Pi=Z Z^{T}\left(\nabla_{\mathbf{n n}}^{2} f+\Lambda\right) Z Z^{T} .
$$

This matrix is necessarily singular, with a nullity at least $n$ (since $Z^{T}$ has an $n$ dimensional null space). To be precise, we have the following.

Proposition 4.6. Let $\mathbf{n}^{*}$ be any constrained stationary point of the discretized free energy $f\left(\mathbf{n}_{1}, \ldots, \mathbf{n}_{n}\right)$ subject to $\left|\mathbf{n}_{j}\right|=1, j=1, \ldots, n$. Then the projected Hessian $H(\mathbf{n})$ (4.18) of the Truncated Newton step (4.19) evaluated at $\mathbf{n}=\mathbf{n}^{*}$ has the form

$$
H\left(\mathbf{n}^{*}\right)=Z Z^{T}\left(\nabla_{\mathbf{n n}}^{2} f\left(\mathbf{n}^{*}\right)+\Lambda\left(\boldsymbol{\lambda}^{*}\right)\right) Z Z^{T}, \quad \boldsymbol{\lambda}^{*}=-B\left(\mathbf{n}^{*}\right)^{T} \nabla_{\mathbf{n}} f\left(\mathbf{n}^{*}\right),
$$

with $\Lambda, B$, and $Z$ as in (3.3), (3.4), and (3.5). As a consequence, the matrix $H\left(\mathbf{n}^{*}\right)$ is singular, and $\mathcal{N}\left(H\left(\mathbf{n}^{*}\right)\right) \supset \mathcal{N}\left(Z^{T}\right)$, which implies that nullity $\left(H\left(\mathbf{n}^{*}\right)\right) \geq n$. If in addition $\mathbf{n}^{*}$ is regular, then

$$
\mathcal{N}\left(H\left(\mathbf{n}^{*}\right)\right)=\mathcal{N}\left(Z^{T}\right) \text { and } \operatorname{nullity}\left(H\left(\mathbf{n}^{*}\right)\right)=n .
$$

Here $\mathcal{N}(M)$ denotes the nullspace of the matrix $M$.

Proof. The facts that the nullspace of $H\left(\mathbf{n}^{*}\right)$ contains the nullspace of $Z^{T}$ and that the nullity of $H\left(\mathbf{n}^{*}\right)$ is greater than or equal to $n$ are clear from the preceding discussion and from the form of $H\left(\mathbf{n}^{*}\right)$ above. It only remains to be shown that if $\mathbf{n}^{*}$ is a regular constrained equilibrium point of $f$, then $\mathcal{N}\left(H\left(\mathbf{n}^{*}\right)\right) \subset \mathcal{N}\left(Z^{T}\right)$, which can be seen as follows. At a regular point $\mathbf{n}^{*}, Z^{T} A Z$ is nonsingular and

$$
\begin{aligned}
\mathbf{p} \in \mathcal{N}\left(H\left(\mathbf{n}^{*}\right)\right) & \Rightarrow Z Z^{T} A Z Z^{T} \mathbf{p}=\mathbf{0} \\
& \Rightarrow Z^{T} Z Z^{T} A Z Z^{T} \mathbf{p}=\mathbf{0} \\
& \Rightarrow Z^{T} A Z Z^{T} \mathbf{p}=\mathbf{0}, \quad \text { since } Z^{T} Z=I \\
& \Rightarrow Z^{T} \mathbf{p}=\mathbf{0}, \quad \text { since } Z^{T} A Z \text { is nonsingular } \\
& \Rightarrow \mathbf{p} \in \mathcal{N}\left(Z^{T}\right) .
\end{aligned}
$$


Therefore, when $\mathbf{n}^{*}$ is regular, the nullspace of $H\left(\mathbf{n}^{*}\right)$ coincides with the nullspace of $Z^{T}$, and the nullity of $H\left(\mathbf{n}^{*}\right)$ is exactly $n$.

Thus the coefficient matrix of the linear system (4.19) is necessarily singular (with a large nullity) at the local minimizers being sought, and the system will be very ill-conditioned in neighborhoods of any such points. Another difficulty with the Truncated Newton Method is that the linear system $H(\mathbf{n}) \mathbf{p}=-\mathbf{G}(\mathbf{n})$ for the projected Newton correction $\mathbf{p}$ is in fact solved by the current approximate discrete director field $\mathbf{n}$. We have the following.

Proposition 4.7. Let $\mathbf{n}$ be a normalized discrete director field $\left(\mathbf{n}=\left(\mathbf{n}_{1}, \ldots, \mathbf{n}_{n}\right)\right.$, $\left.\left|\mathbf{n}_{1}\right|=\cdots=\left|\mathbf{n}_{n}\right|=1\right), f(\mathbf{n})$ a discrete free energy, and $\mathbf{G}(\mathbf{n})$ and $H(\mathbf{n})$ the projected gradient and Hessian of the Truncated Newton Method, (4.16) and (4.18) respectively. Then the linear system for the Truncated Newton step $H(\mathbf{n}) \mathbf{p}=-\mathbf{G}(\mathbf{n})$ is solved by $\mathbf{p}=\mathbf{n}$ :

$$
H(\mathbf{n}) \mathbf{n}=-\mathbf{G}(\mathbf{n}) .
$$

Proof. This can be verified directly:

$$
Z^{T} \mathbf{n}=\mathbf{0} \Rightarrow H \mathbf{n}=-H_{2} \mathbf{n}=-\left[\begin{array}{c}
\vdots \\
\left(\mathbf{G}_{i} \cdot \mathbf{n}_{i}\right) \mathbf{n}_{i}+\left(\mathbf{n}_{i} \cdot \mathbf{n}_{i}\right) \mathbf{G}_{i} \\
\vdots
\end{array}\right]=-\left[\begin{array}{c}
\vdots \\
\mathbf{G}_{i} \\
\vdots
\end{array}\right]=-\mathbf{G},
$$

since $\mathbf{G}_{i} \cdot \mathbf{n}_{i}=0$ and $\mathbf{n}_{i} \cdot \mathbf{n}_{i}=1, i=1, \ldots, n$. This can also be seen from (63) of [8], by directly verifying that $H[\mathbf{m}] \mathbf{m}=-G[\mathbf{m}]$ (in the notation of that paper).

Thus the linear system for the Truncated Newton step (4.19) is always consistent and always admits the solution $\mathbf{p}=\mathbf{n}$. If $H$ is nonsingular, then $\mathbf{p}=\mathbf{n}$ is the only solution. The difficulty with this is that the vector $\mathbf{p}=\mathbf{n}$ does not provide a descent direction to use in a line search: it is in the normal space to the constraint manifold at $\mathbf{n}$ and has no component in the tangent space. Both of these properties (singularity of $H\left(\mathbf{n}^{*}\right)$ and $\mathbf{p}=\mathbf{n}$ solution of $H(\mathbf{n}) \mathbf{p}=-\mathbf{G}(\mathbf{n})$ ) are confirmed by numerical experiments in the Supplementary Materials.

One must keep in mind that the Truncated Newton step (4.19) is just a part of an energy minimization algorithm. As described in [8], this system is solved approximately via a Preconditioned Conjugate Gradient method, and the approximate solution vector $\mathbf{p}$ is tested to see if it is a descent direction for the discretized LandauLifshitz free energy. If it is, then it is used in the subsequent line search; if not, another $\mathbf{p}$ is used (the steepest-descent direction $\mathbf{p}=-\mathbf{G}(\mathbf{n})$, one would suppose). Codes based upon the Truncated Newton Method energy minimization approach have been used to compute interesting domain patterns and structures in thin magnetic films $[5,6,7,8]$, and it is indicated in those references that the same solutions have also been obtained by separate codes using a different approach (time relaxation to steady state). We can only assume that in the energy minimization code, either the approximately computed solution to $H(\mathbf{n}) \mathbf{p}=-\mathbf{G}(\mathbf{n})$ has enough of a component in the tangent space for it to be used in a line search or that the steepest-descent direction is chosen instead in the logic of the code at that stage. It does not seem possible for the Truncated Newton Method, as described in the references above, to be locally quadratically convergent, and we are not aware of any analysis or numerical benchmarking in support of that. The problems of interest to us are saddle-point problems, in any event. 

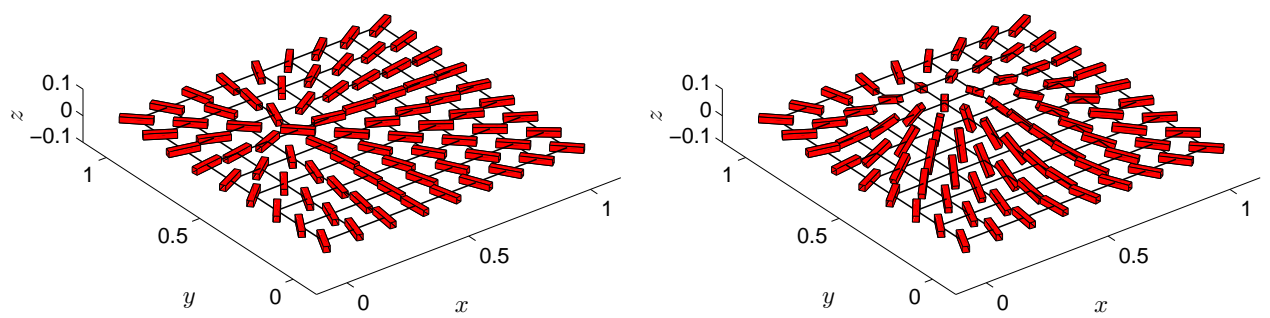

FIG. 5.1. Equilibrium director fields of Harmonic Mapping Problem (5.1): planar disclination line (left, locally unstable), upward "escape" solution (right, locally stable). Not displayed is a downward "escape" solution, which is the mirror image with respect to the xy plane of the pictured upward "escape" solution.

5. Numerical examples. We contrast some numerical aspects of the basic Newton Method and the Renormalized Newton Method on a model problem in two space dimensions. The problem admits multiple solutions, equilibrium director field solutions with defects as well as solutions that are defect free.

5.1. Model problem and discretization. We consider the "Harmonic Mapping Problem" associated with an equal-elastic-constant free energy functional, with no electric or magnetic field, rescaled to the unit square, and subject to the boundary conditions of a line disclination (as in (4.12)):

$$
\begin{gathered}
\min _{|\boldsymbol{n}|=1} \mathcal{F}[\boldsymbol{n}], \quad \mathcal{F}[\boldsymbol{n}]=\frac{1}{2} \int_{\Omega}|\nabla \boldsymbol{n}|^{2} d A=\frac{1}{2} \int_{\Omega}\left[|\nabla u|^{2}+|\nabla v|^{2}+|\nabla w|^{2}\right] d A \\
\boldsymbol{n}=u(x, y) \boldsymbol{e}_{x}+v(x, y) \boldsymbol{e}_{y}+w(x, y) \boldsymbol{e}_{z}, \quad \Omega=(0,1)^{2} \\
\boldsymbol{n}=\boldsymbol{n}_{\mathrm{b}} \quad \text { on } \partial \Omega, \quad \boldsymbol{n}_{\mathrm{b}}(x, y)=\frac{\left(x-x_{\mathrm{d}}\right) \boldsymbol{e}_{x}+\left(y-y_{\mathrm{d}}\right) \boldsymbol{e}_{y}}{\sqrt{\left(x-x_{\mathrm{d}}\right)^{2}+\left(y-y_{\mathrm{d}}\right)^{2}}} .
\end{gathered}
$$

The defect location is taken to be $\left(x_{\mathrm{d}}, y_{\mathrm{d}}\right)=(1 / 3,2 / 3)$ in the examples computed below. For this combination of geometry and boundary conditions, we anticipate (and find) three equilibrium solutions: an $x y$-planar line disclination and a pair of solutions that are mirror images of each other (across the $x y$ plane) and which avoid the singularity of the planar solution by "escaping into the third dimension" - see Fig. 5.1. The two "escape" solutions are locally stable - appropriate criteria for local stability for discretizations of such problems are derived in $[9,10]$ and discussed below. The disclination line solution is technically inadmissible for an Oseen-Frank free energy - the integral functional is divergent, as was pointed out in $\S 4.2$ - but it satisfies the Euler-Lagrange equations away from the defect point and is useful for numerical illustrations.

This model problem is discretized with bilinear finite elements on a uniform mesh, in the notation

$$
\mathbf{n}_{i j} \approx \boldsymbol{n}\left(x_{i}, y_{j}\right), \quad x_{i}=i h, y_{j}=j h, \quad i, j=0, \ldots, n, \quad h=1 / n .
$$

A four-point, nodal quadrature rule is used,

$$
\int_{y_{j}}^{y_{j+1}} \int_{x_{i}}^{x_{i+1}} W(x, y) d x d y \approx \frac{h^{2}}{4}\left[W_{i j}+W_{i+1, j}+W_{i, j+1}+W_{i+1, j+1}\right]
$$


resulting in the discretized free energy

$$
\begin{array}{r}
f(\mathbf{n})=\frac{1}{4} \sum_{i, j=0}^{n-1}\left[\left|\mathbf{n}_{i+1, j}-\mathbf{n}_{i j}\right|^{2}+\left|\mathbf{n}_{i+1, j+1}-\mathbf{n}_{i, j+1}\right|^{2}\right. \\
\left.+\left|\mathbf{n}_{i, j+1}-\mathbf{n}_{i j}\right|^{2}+\left|\mathbf{n}_{i+1, j+1}-\mathbf{n}_{i+1, j}\right|^{2}\right] .
\end{array}
$$

In the above expression, appropriate boundary values are used for $\mathbf{n}_{i j}$ at all boundary nodes. The constrained equilibrium equations derive from the Lagrangian, as in (3.1), and take the form

$$
\begin{gathered}
\nabla_{\mathbf{n}_{i j}} L=\nabla_{\mathbf{n}_{i j}} f+\lambda_{i j} \mathbf{n}_{i j}=-\Delta_{h} \mathbf{n}_{i j}+\lambda_{i j} \mathbf{n}_{i j} \\
\Delta_{h} \mathbf{n}_{i j}:=\mathbf{n}_{i, j-1}+\mathbf{n}_{i-1, j}+\mathbf{n}_{i+1, j}+\mathbf{n}_{i, j+1}-4 \mathbf{n}_{i j},
\end{gathered}
$$

giving the difference equations (from $\nabla_{\mathbf{n}} L=\mathbf{0}$ )

$$
\begin{aligned}
-\Delta_{h} \mathbf{n}_{i j}+\lambda_{i j} \mathbf{n}_{i j} & =\mathbf{0}, \quad\left|\mathbf{n}_{i j}\right|=1, \quad i, j=1, \ldots, n-1 \\
\mathbf{n}_{i j} & =\mathbf{n}_{i j}^{\mathrm{b}}, \quad i \text { or } j=0 \text { or } n .
\end{aligned}
$$

This scheme is readily seen to be $O\left(h^{2}\right)$ consistent with the Euler-Lagrange equations of the continuous problem, which are usually written

$$
\Delta \boldsymbol{n}+\lambda \boldsymbol{n}=\mathbf{0}, \quad|\boldsymbol{n}|=1 \text { in } \Omega, \quad \boldsymbol{n}=\boldsymbol{n}_{\mathrm{b}} \text { on } \partial \Omega,
$$

although the signs and scalings of the Lagrange multipliers are different. The Lagrange multiplier field for a solution to (5.4) above can be derived using $\boldsymbol{n} \cdot \boldsymbol{n}=1$ and is given by

$$
\lambda=-\Delta \boldsymbol{n} \cdot \boldsymbol{n}=|\nabla \boldsymbol{n}|^{2},
$$

which is $O(1)$ away from defects but $O\left(1 / r^{2}\right)$ near them-here $r$ is the distance to the defect point.

Numerical experiments were conducted in Matlab using both the basic Newton scheme ( $\S 3$ above) and the Renormalized Newton scheme ( $\$ 4$ above) for this discretized model problem. Linear systems were solved by the Matlab backslash operator using sparse direct numerical linear algebra. The blocks of the Hessian were built as in $\S 3$, with the $A_{0}$ matrix of (3.3) here given by

$$
A_{0}=\nabla_{\mathbf{n n}}^{2} f=-\left[\begin{array}{lll}
\Delta_{h} & & \\
& \Delta_{h} & \\
& & \Delta_{h}
\end{array}\right]
$$

(with $\Delta_{h}$ the matrix of the 2-D discrete Laplacian associated with the difference operator of $(5.2))$ with respect to the ordering

$$
\mathbf{n}=\left(u_{1}, \ldots, u_{N}, v_{1}, \ldots, v_{N}, w_{1}, \ldots, w_{N}\right), \quad N:=(n-1)^{2} .
$$

Initial guesses were constructed by blending the true continuous line-disclination solution with a vertical field in the interior:

$$
\mathbf{n}_{i j}^{\mathrm{init}}=\frac{(1-|\alpha|) \mathbf{n}_{i j}^{\mathrm{b}}-\alpha \boldsymbol{e}_{z}}{\left|(1-|\alpha|) \mathbf{n}_{i j}^{\mathrm{b}}-\alpha \boldsymbol{e}_{z}\right|}, \quad i, j=1, \ldots, n-1, \quad-1 \leq \alpha \leq 1,
$$

with $\mathbf{n}_{\mathrm{b}}$ as in (5.1). Positive $\alpha$ 's correspond roughly to upward escape, while negative $\alpha$ 's correspond to downward escape. For $\alpha \in[-0.3,0.3]$, we normally obtained convergence to the planar solution; while with $\alpha \approx 0.6$ or -0.6 , we normally obtained the upward or downward "escape" solution respectively. The values $\alpha=0.3$ and $\alpha=0.6$ were used in most of the experiments below. 
TABLE 5.1

Convergence behavior of the basic Newton Method versus the Renormalized Newton Method for the model Harmonic Mapping Problem (5.1), discretized as in (5.3), with $n=128\left(N=127^{2}\right.$ interior nodes). Final computed solution is the planar line disclination as in Fig. 5.1 left (with a defect at the point $\left.\left(x_{d}, y_{d}\right)=(1 / 3,2 / 3)\right)$, from the initial guess (5.6) with $\alpha=0.3$.

\begin{tabular}{|r|cccc|cc|}
\hline & \multicolumn{5}{|c|}{ Newton } & \multicolumn{2}{c|}{ Renormalized } \\
\cline { 2 - 7 } iter & $\left\|\nabla_{\mathbf{n}} L\right\|_{\infty}$ & $\left\|\nabla_{\boldsymbol{\lambda}} L\right\|_{\infty}$ & $\|\boldsymbol{\delta} \mathbf{n}\|_{\infty}$ & $\|\boldsymbol{\delta} \boldsymbol{\lambda}\|_{\infty}$ & $\left\|Z^{T} \nabla_{\mathbf{n}} f\right\|_{\infty}$ & $\|\boldsymbol{\delta} \mathbf{n}\|_{\infty}$ \\
\hline 1 & $9.71(-01)$ & $2.22(-16)$ & $1.28(+00)$ & $1.53(+00)$ & $1.06(+00)$ & $1.28(+00)$ \\
2 & $7.15(-01)$ & $9.75(-01)$ & $1.53(+00)$ & $3.26(-01)$ & $3.38(-01)$ & $6.64(-01)$ \\
3 & $1.73(-01)$ & $1.21(+00)$ & $8.66(-01)$ & $7.55(-01)$ & $4.44(-01)$ & $5.42(-01)$ \\
4 & $4.23(-01)$ & $4.46(-01)$ & $3.23(-01)$ & $5.89(-01)$ & $1.64(-01)$ & $2.19(-01)$ \\
5 & $1.07(-01)$ & $9.99(-02)$ & $1.57(-01)$ & $1.25(-01)$ & $6.35(-02)$ & $6.43(-02)$ \\
6 & $1.81(-02)$ & $1.26(-02)$ & $1.85(-02)$ & $2.10(-02)$ & $1.86(-03)$ & $1.49(-03)$ \\
7 & $3.90(-04)$ & $1.96(-04)$ & $4.25(-04)$ & $2.56(-04)$ & $4.32(-06)$ & $1.50(-05)$ \\
8 & $1.09(-07)$ & $1.03(-07)$ & $1.55(-07)$ & $2.47(-07)$ & $5.55(-11)$ & $3.05(-11)$ \\
9 & $3.82(-14)$ & $1.24(-14)$ & $4.56(-14)$ & $1.58(-14)$ & $1.00(-15)$ & $2.10(-15)$ \\
10 & $9.97(-16)$ & $2.22(-16)$ & $3.86(-15)$ & $1.33(-15)$ & $9.07(-16)$ & $1.74(-15)$ \\
11 & $9.60(-16)$ & $2.22(-16)$ & $3.00(-15)$ & $1.33(-15)$ & $1.02(-15)$ & $2.50(-15)$ \\
\hline
\end{tabular}

5.2. Numerical results. In the absence of any electric or magnetic field, the basic Newton step (3.2) takes the form

$$
\left[\begin{array}{cc}
A & B \\
B^{T} & O
\end{array}\right]\left[\begin{array}{l}
\boldsymbol{\delta} \mathbf{n} \\
\boldsymbol{\delta} \boldsymbol{\lambda}
\end{array}\right]=-\left[\begin{array}{c}
\nabla_{\mathbf{n}} L \\
\nabla_{\boldsymbol{\lambda}} L
\end{array}\right], \quad \mathbf{n} \leftarrow \mathbf{n}+\boldsymbol{\delta} \mathbf{n}, \quad \boldsymbol{\lambda} \leftarrow \boldsymbol{\lambda}+\boldsymbol{\delta} \boldsymbol{\lambda},
$$

while the Renormalized Newton step becomes

$$
Z^{T} A Z \mathbf{p}=-Z^{T} \nabla_{\mathbf{n}} f, \quad \boldsymbol{\delta} \mathbf{n}=Z \mathbf{p}, \quad \mathbf{n}_{i j} \leftarrow \frac{\mathbf{n}_{i j}+\boldsymbol{\delta} \mathbf{n}_{i j}}{\left|\mathbf{n}_{i j}+\delta \mathbf{n}_{i j}\right|} .
$$

Here $A=A_{0}+\Lambda(\boldsymbol{\lambda})$ as in (3.3), with $\boldsymbol{\lambda}$ given by the current approximate $\boldsymbol{\lambda}$ for the Newton step versus $\boldsymbol{\lambda}=-B(\mathbf{n})^{T} \nabla_{\mathbf{n}} f(\mathbf{n})$ for the Renormalized Newton step. Both iterations were started from the same initial guess, as in (5.6), with $\boldsymbol{\lambda}_{\text {init }}=$ $-B\left(\mathbf{n}_{\text {init }}\right)^{T} \nabla_{\mathbf{n}} f\left(\mathbf{n}_{\text {init }}\right)$ used to initialize the Lagrange multipliers for the basic Newton iteration - thus both $\mathbf{n}$ and $\boldsymbol{\lambda}$ coincide entering the main loops for both iterative solvers. Representative data for the convergence behavior of the two methods are given in Tables 5.1 and 5.2 with $n=128$ for both the planar disclination-line solution (which is locally unstable) and the (upward) "escape" solution (which is locally stable). The tables indicate that the Renormalized Newton Method converges a little more rapidly (normally reaching target tolerances in one or two fewer iterations than the basic Newton scheme) and that the convergence behavior of both iterations is essentially the same for solution fields with defects as it is for those without defects.

The Renormalized Newton Method was also found to be a little more "robust," in the sense that it would sometimes converge from an initial guess from which the basic Newton iteration failed. This is illustrated in Table 5.3, which also gives the number of iterations required for each to achieve machine attainable accuracy from the same initial guess. The numbers to put in such a table are somewhat subjective, since the last iteration or two before convergence completely stalls typically only make incremental progress. For example, for the data in Tables 5.1 and 5.2, we would say that the Newton scheme is fully converged after 10 iterations for the planar solution and 11 iterations for the escaped solution, while the Renormalized Newton scheme takes 9 and 8 iterations respectively. 
TABLE 5.2

Convergence behavior of the basic Newton Method versus the Renormalized Newton Method for the model Harmonic Mapping Problem (5.1), discretized as in (5.3), with $n=128\left(N=127^{2}\right.$ interior nodes). Final computed solution is the upward "escape" solution as in Fig. 5.1 right (which is defect free), from the initial guess (5.6) with $\alpha=0.6$.

\begin{tabular}{|r|cccc|cc|}
\hline & \multicolumn{5}{|c|}{ Newton } & \multicolumn{2}{c|}{ Renormalized } \\
\cline { 2 - 7 } iter & $\left\|\nabla_{\mathbf{n}} L\right\|_{\infty}$ & $\left\|\nabla_{\boldsymbol{\lambda}} L\right\|_{\infty}$ & $\|\boldsymbol{\delta} \mathbf{n}\|_{\infty}$ & $\|\boldsymbol{\delta} \boldsymbol{\lambda}\|_{\infty}$ & $\left\|Z^{T} \nabla_{\mathbf{n}} f\right\|_{\infty}$ & $\|\boldsymbol{\delta} \mathbf{n}\|_{\infty}$ \\
\hline 1 & $1.24(+00)$ & $1.67(-16)$ & $1.44(+00)$ & $2.98(+00)$ & $1.54(+00)$ & $1.44(+00)$ \\
2 & $5.84(-01)$ & $2.23(+00)$ & $9.98(-01)$ & $1.85(+00)$ & $2.97(-01)$ & $1.33(+00)$ \\
3 & $6.64(-01)$ & $1.03(+00)$ & $9.18(-01)$ & $8.07(-01)$ & $1.89(-01)$ & $3.06(-01)$ \\
4 & $7.18(-01)$ & $9.57(-01)$ & $7.83(-01)$ & $6.11(-01)$ & $3.70(-03)$ & $4.71(-02)$ \\
5 & $4.58(-01)$ & $7.76(-01)$ & $5.56(-01)$ & $2.06(-01)$ & $1.15(-05)$ & $9.92(-04)$ \\
6 & $1.02(-01)$ & $2.84(-01)$ & $1.74(-01)$ & $1.20(-01)$ & $1.94(-09)$ & $8.67(-07)$ \\
7 & $2.08(-02)$ & $3.02(-02)$ & $4.20(-02)$ & $4.43(-02)$ & $1.74(-15)$ & $2.04(-13)$ \\
8 & $1.73(-03)$ & $1.17(-03)$ & $2.61(-03)$ & $2.11(-03)$ & $9.43(-16)$ & $5.66(-15)$ \\
9 & $5.51(-06)$ & $6.87(-06)$ & $1.20(-05)$ & $8.70(-06)$ & $8.91(-16)$ & $4.51(-15)$ \\
10 & $1.04(-10)$ & $1.33(-10)$ & $1.82(-10)$ & $2.01(-10)$ & $8.85(-16)$ & $4.83(-15)$ \\
11 & $8.84(-16)$ & $2.22(-16)$ & $3.95(-15)$ & $1.18(-15)$ & & \\
12 & $1.02(-15)$ & $2.22(-16)$ & $3.95(-15)$ & $1.21(-15)$ & & \\
\hline
\end{tabular}

TABLE 5.3

Number of iterations required to achieve machine attainable accuracy by the basic Newton Method versus the Renormalized Newton Method for the model Harmonic Mapping Problem (5.1), discretized as in (5.3). Initial guesses for both the planar disclination-line solution (with defect at $\left(x_{d}, y_{d}\right)=(1 / 3,2 / 3)$ ) and the upward "escape" solution (which is defect free) were given by (5.6), with $\alpha=0.3$ and $\alpha=0.6$ respectively. With $\alpha=0.3$, for $n=16$ and $n=32$, the basic Newton iteration failed to converge; while for $n=4$, the Renormalized Newton Method converged to the "wrong solution" (the downward "escape" solution instead of the planar solution). With $\alpha=0.6$, for $n=64$, the Newton scheme again failed to converge.

\begin{tabular}{|r|cc|cc|}
\hline & \multicolumn{2}{|c|}{ planar solution $(\alpha=0.3)$} & \multicolumn{2}{c|}{ escape solution $(\alpha=0.6)$} \\
\cline { 2 - 5 }$n$ & Newton & $\begin{array}{c}\text { RN } \\
\text { iterations }\end{array}$ & $\begin{array}{c}\text { Newton } \\
\text { iterations }\end{array}$ & $\begin{array}{c}\text { RN } \\
\text { iterations }\end{array}$ \\
\hline 4 & 16 & - & 7 & 6 \\
8 & 7 & 7 & 7 & 6 \\
16 & - & 8 & 7 & 6 \\
32 & - & 11 & 12 & 7 \\
64 & 11 & 9 & -11 & 7 \\
128 & 10 & 9 & 11 & 8 \\
256 & 8 & 9 & 9 & 8 \\
512 & 9 & 9 & 9 & 8 \\
\hline
\end{tabular}

The size of the Newton system (number of unknowns) is $4 N$ (with $N=(n-1)^{2}$ ), whereas the size of the Renormalized Newton system is $2 N$. The sparsities of the coefficient matrices are comparable. As a consequence, the Renormalized Newton Method requires less time per iteration when the linear systems are solved by direct methods, as we have done in these experiments. Table 5.4 compares the sizes of the two systems and the time per iteration. This is a casual comparison, using elapsed clock time obtained via Matlab's tic-toc functions (total time for the main loop divided by the total number of times through the loop). The runs were done on a laptop with a $2.93 \mathrm{GHz}$ dual-core processor and $8 \mathrm{~GB}$ memory.

For problems for which iterative methods are necessary, we would use MINRES for both the basic Newton Method as well as the Renormalized Newton Methodthe coefficient matrix for the Newton scheme (5.7) is highly indefinite, and $Z^{T} A Z$ is indefinite on branches of locally unstable solutions (which one often needs to follow in 
TABLE 5.4

System size and time per iteration for the basic Newton Method and the Renormalized Newton Method for representative runs for the Harmonic Mapping Problem (5.1), discretized as in (5.3). Here $n=$ number of mesh cells in one direction; $N=(n-1)^{2}=$ number of interior nodes; size $=$ total number of unknowns ( $4 N$ for Newton, $2 N$ for Renormalized Newton); time $=$ time per iteration (in seconds, from tic-toc, total time for execution of main loop divided by number of times through the loop).

\begin{tabular}{|rr|rc|rc|}
\hline & & \multicolumn{2}{|c|}{ Newton } & \multicolumn{2}{c|}{ Renormalized } \\
\cline { 3 - 6 }$n$ & \multicolumn{1}{|c|}{$N$} & \multicolumn{1}{|c|}{ size } & time & \multicolumn{1}{c|}{ size } & time \\
\hline 4 & 9 & 36 & $3.50(-3)$ & 18 & $3.00(-3)$ \\
8 & 49 & 196 & $5.50(-3)$ & 98 & $5.00(-3)$ \\
16 & 225 & 900 & $1.64(-2)$ & 450 & $1.24(-2)$ \\
32 & 961 & 3,844 & $6.73(-2)$ & 1,922 & $4.42(-2)$ \\
64 & 3,969 & 15,876 & $4.52(-1)$ & 7,938 & $2.33(-1)$ \\
128 & 16,129 & 64,516 & $2.71(+0)$ & 32,258 & $1.21(+0)$ \\
256 & 65,025 & 260,100 & $1.83(+1)$ & 130,050 & $6.71(+0)$ \\
512 & 261,121 & $1,044,484$ & $1.66(+2)$ & 522,242 & $3.55(+1)$ \\
\hline
\end{tabular}

TABLE 5.5

1-norm condition numbers for the coefficient matrices of the basic Newton Method (M in (5.8)) and the Renormalized Newton Method $\left(Z^{T} A Z\right)$ on the fully converged planar defect solution (Fig. 5.1 left) and the (upward) "escape" defect-free solution (Fig. 5.1 right) of the Harmonic Mapping Problem (5.1), discretized as in (5.3). Condition numbers were estimated using Matlab's condest() function.

\begin{tabular}{|r|cc|cc|}
\hline \multirow{2}{*}{$n$} & \multicolumn{2}{|c|}{ defect solution } & \multicolumn{2}{c|}{ defect-free solution } \\
\cline { 2 - 5 } & $\operatorname{cond}(M)$ & $\operatorname{cond}\left(Z^{T} A Z\right)$ & $\operatorname{cond}(M)$ & $\operatorname{cond}\left(Z^{T} A Z\right)$ \\
\hline 4 & $7.07(+01)$ & $1.79(+01)$ & $8.66(+01)$ & $2.21(+01)$ \\
8 & $1.68(+02)$ & $5.57(+01)$ & $1.30(+02)$ & $8.93(+01)$ \\
16 & $3.01(+02)$ & $2.55(+02)$ & $4.55(+02)$ & $3.47(+02)$ \\
32 & $1.57(+03)$ & $1.22(+03)$ & $1.71(+03)$ & $1.39(+03)$ \\
64 & $3.79(+04)$ & $3.37(+04)$ & $6.77(+03)$ & $5.72(+03)$ \\
128 & $2.13(+04)$ & $1.89(+04)$ & $2.70(+04)$ & $1.89(+04)$ \\
256 & $7.90(+04)$ & $7.02(+04)$ & $1.08(+05)$ & $8.81(+04)$ \\
512 & $3.56(+05)$ & $3.17(+05)$ & $4.31(+05)$ & $3.52(+05)$ \\
\hline
\end{tabular}

numerical bifurcation analysis). The Newton system can be optimally preconditioned using the approach of $[14, \S 6]$. Optimal preconditioners for $Z^{T} A Z$ will be the subject of subsequent work [10].

The condition numbers of the coefficient matrices

$$
M=\left[\begin{array}{cc}
A & B \\
B^{T} & O
\end{array}\right] \quad \text { vs } \quad Z^{T} A Z
$$

were found to be comparable and to scale as expected for a discretization of a secondorder PDE problem:

$$
\operatorname{cond}(M), \operatorname{cond}\left(Z^{T} A Z\right)=O\left(1 / h^{2}\right)=O\left(n^{2}\right) .
$$

The variation in the condition numbers was also negligible when comparing solutions with defects to those without. See Table 5.5, where the 1-norm condition numbers were estimated using Matlab's condest() function.

For both schemes, one area in which a difference can be seen in the behavior on the defect director field (the locally unstable planar solution) versus the defect-free equilibrium director fields (the locally stable up/down "escape" solutions) is in the values of the Lagrange multipliers. The way this problem has been scaled, the discrete 
TABLE 5.6

Least and greatest discrete Lagrange multipliers for fully converged planar solution (disclination line, Fig. 5.1 left) versus "escape" solution (defect-free upward "escape" solution, Fig. 5.1 right) of the Harmonic Mapping Problem (5.1), discretized as in (5.3).

\begin{tabular}{|r|cc|cc|}
\hline & \multicolumn{2}{|c|}{ planar solution } & \multicolumn{2}{c|}{ "escape" solution } \\
\cline { 2 - 5 }$n$ & $\min \lambda_{i j}$ & $\max \lambda_{i j}$ & $\min \lambda_{i j}$ & $\max \lambda_{i j}$ \\
\hline 4 & $-2.47(+0)$ & $-2.05(-1)$ & $-1.76(+0)$ & $-2.97(-1)$ \\
8 & $-2.28(+0)$ & $-2.71(-2)$ & $-5.09(-1)$ & $-3.62(-2)$ \\
16 & $-2.20(+0)$ & $-5.38(-3)$ & $-1.33(-1)$ & $-5.97(-3)$ \\
32 & $-2.20(+0)$ & $-1.21(-3)$ & $-3.33(-2)$ & $-1.25(-3)$ \\
64 & $-2.18(+0)$ & $-2.88(-4)$ & $-8.35(-3)$ & $-2.90(-4)$ \\
128 & $-2.17(+0)$ & $-7.03(-5)$ & $-2.09(-3)$ & $-7.04(-5)$ \\
256 & $-2.17(+0)$ & $-1.74(-5)$ & $-5.22(-4)$ & $-1.74(-5)$ \\
512 & $-2.17(+0)$ & $-4.32(-6)$ & $-1.30(-4)$ & $-4.32(-6)$ \\
\hline
\end{tabular}

Lagrange multipliers satisfy

$$
\lambda_{i j} \approx-h^{2} * \lambda\left(x_{i}, y_{j}\right)
$$

where $\lambda(x, y)$ is the Lagrange multiplier field of the continuous Euler-Lagrange equation (5.4). As observed in the previous subsection, $\lambda=|\nabla \boldsymbol{n}|^{2}$ for solutions of (5.4), and so by virtue of (4.14), we have

$$
\lambda_{i j}= \begin{cases}O\left(h^{2}\right), & \text { for defect-free solutions } \\ O\left(h^{2}\left(1+1 / r^{2}\right)\right), & \text { for director fields with defects. }\end{cases}
$$

Thus the discrete Lagrange multipliers should be $O\left(h^{2}\right)$ throughout the domain for the "escape" solutions, while they will attain maximal $O(1)$ values on the nodes of the mesh cell containing the defect point for the planar solutions. This is indeed what is observed, as demonstrated in Table 5.6.

5.3. Discussion. The performance of both the basic Newton Method and the Renormalized Newton Method was very satisfactory on these model test problems. It is perhaps a little surprising that there wasn't more of a difference between the runs with the defect solutions versus those with the defect-free solutions: the condition numbers and convergence rates and the like were all quite comparable. The Renormalized Newton Method displayed some modest advantages: more robust with respect to obtaining convergence from crude initial guesses, fewer iterations to achieve convergence tolerances in general (one or two per run, typically), and less execution time (roughly half the time of the basic Newton Method for realistic $n$ 's, improving as $n$ increases). For the applications of interest to us (involving parameter continuation, path following, numerical bifurcation and phase analysis), which require the repeated solution of such systems, these incremental advantages can lead to appreciable gains in efficiency. For applications in three space dimensions, iterative methods would be required for the numerical linear algebra, and preconditioned MINRES would be used. Optimal preconditioners for the basic Newton Method are known [14]. Preconditioners for the Renormalized Newton Method are under development [10].

It is worthwhile to comment at this point on the spectral properties of the matrices that arise in these methods:

$$
A=A_{0}+\Lambda, \quad M=\left[\begin{array}{cc}
A & B \\
B^{T} & O
\end{array}\right], \quad \text { and } \quad Z^{T} A Z .
$$


As previously noted, $M$ is nonsingular if and only if $Z^{T} A Z$ is nonsingular (assuming $B$ and $Z$ are constructed as in $\S 3$ and all of the discrete directors are nonzero). Local stability of solutions of such problems (including cases with coupled electric fields) is discussed in [9, 10]. For the model Harmonic Mapping Problem used in the numerical experiments of this section, it is simply a matter of considering the minimum eigenvalue of $Z^{T} A Z$ :

$$
\begin{aligned}
& \lambda_{\min }\left(Z^{T} A Z\right)>0 \Rightarrow \text { locally stable } \\
& \lambda_{\min }\left(Z^{T} A Z\right)<0 \Rightarrow \text { locally unstable. }
\end{aligned}
$$

The $A$ matrix is symmetric, and for an unstable solution, it is necessarily indefinite (by virtue of the above). For a stable solution, $A$ need not be positive definite or even nonsingular, and for our numerical experiments with locally stable solutions, $A$ was found to be positive semi-definite but singular with a zero eigenvalue of multiplicity at least three. The $M$ matrix is $4 N \times 4 N$ symmetric but highly indefinite. On locally stable solutions, it has $3 N$ positive eigenvalues and $N$ negative ones; while on unstable solutions, it has more than $N$ negative eigenvalues. For our numerical experiments, $Z^{T} A Z$ was indeed found to be positive definite on stable solutions. On unstable solutions, it was nonsingular but indefinite, with one negative eigenvalue for small values of $n$ and two negative eigenvalues for large values of $n$.

A crossover in the behavior of the matrices was observed in going from $n=64$ to $n=128$. For $n=4,8, \ldots, 64$, the $A$ matrix has a zero eigenvalue of multiplicity three, and $Z^{T} A Z$ has one negative eigenvalue; while for $n=128,256$, and 512, the $A$ matrix appears to have a slightly higher-dimensional null space (five or six, more likely the latter), and $Z^{T} A Z$ has two negative eigenvalues. One can also detect the approach of this crossover in Table 5.5, in which the condition numbers of both $M$ and $Z^{T} A Z$ are larger than the trends would suggest for $n=64$ on the planar, disclination-line solution. Numerical experiments on aspects of the Truncated Newton Method are reported in $\S \mathrm{S} 3$ of the Supplementary Material.

6. Conclusion. We have introduced and studied a prototype director model for the equilibrium orientational configuration in a liquid crystal material, focusing on the commonly occurring case of a coupled electric-field interaction. The prototype model embodies the essential features of models for realistic experiments and device simulations. It also shares similar features with the Landau-Lifshitz model for the magnetization in a ferromagnetic material, and the relationship between the two has been discussed. The equilibrium equations associated with discretizations of the liquid crystal model have a double saddle-point structure, arising from the pointwise unitvector constraints on the components of the director field and from the nature of the coupling between the director field and the local electric field. This paper complements [21], where we have proposed a preconditioned nullspace method as an effective way to solve the associated Lagrange-Newton equations, and the basic ideas of that paper have been reviewed here. The main results here have been the introduction of a modified version of Newton's method (which we refer to as the "Renormalized Newton Method") that takes advantage of the special structure of the problem and the proof that this method is locally quadratically convergent.

The Renormalized Newton scheme has two key features: eliminating the Lagrange multipliers (by least-squares approximations) and renormalizing the local directors at each iterative step. The resulting outer iteration only involves the director and electrostatic variables and remains on the constraint manifold at each stage. In addition 
to proving quadratic convergence of our method (whenever the basic global Newton method is so), we have also presented numerical experiments on a model problem in two space dimensions comparing the basic Newton Method and the Renormalized Newton Method. The test problem admits three different solutions, one with a defect (a line disclination) and two that are defect free. The convergence behavior of neither scheme was affected by the presence of the defect. In general, the Renormalized Newton scheme demonstrated several advantages over the basic Newton scheme: smaller system size, less time per iteration, fewer iterations required to achieve convergence tolerances, and convergence from some crude initial guesses from which the basic Newton scheme failed to converge.

The Renormalized Newton Method bears some resemblance to the Truncated Newton Method of computational micromagnetics, and so we have carefully compared and contrasted the two. This revealed some anomalies of the Truncated Newton approach. In particular, the linear system for the basic Truncated Newton step does not appear to yield solutions that provide descent directions for the discretized free energy being minimized, and the coefficient matrix of this system is necessarily singular at the solutions being sought.

It is also common in computational micromagnetics to use numerical solutions of the time-dependent Landau-Lifshitz-Gilbert equations to obtain desired steady state minimizers of the Landau-Lifshitz free energy, and schemes with provable exponential convergence in time can be found in [20]. The application domain of interest to us (and main motivation for this work) is concerned with numerical bifurcation and phase analysis, for which it is necessary to be able to follow branches of unstable solutions (as well as branches of stable solutions). While time-stepping methods are often useful for obtaining starting points on solution branches, they can't be used systematically for full continuation and path following in general (because of the inability to compute unstable solutions with them).

The main thrust of this paper (and its predecessor [21]) is effective numerical techniques for dealing with pointwise unit-vector constraints in discretizations of equilibrium problems of the type found in liquid crystal director modeling. The ease with which the tangent spaces and normal spaces of the constraint manifold can be characterized and constructed for these particular types of constraints makes nullspace (reduced Hessian) methods very natural and effective and leads to a structure that can be further exploited. The ideas here are not tied to a particular problem or discretization approach. The techniques should be useful in a variety of settings in which such constraints appear, including computational micromagnetics.

\section{REFERENCES}

[1] F. Alouges, A new algorithm for computing liquid crystal stable configurations: the harmonic mapping case, SIAM J. Numer. Anal., 34 (1997), pp. 1708-1726.

[2] M. Benzi, G. H. Golub, And J. Liesen, Numerical solutions of saddle point problems, Acta Numerica, 14 (2005), pp. 1-137.

[3] R. Cohen, S.-Y. Lin, AND M. Luskin, Relaxation and gradient methods for molecular orientation in liquid crystals, Comput. Phys. Comm., 53 (1989), pp. 455-465.

[4] J. E. Dennis, JR. And R. B. Schnabel, Numerical Methods for Unconstrained Optimization and Nonlinear Equations, Prentice-Hall, Englewood Cliffs, 1983.

[5] C. J. García-Cervera, One-dimensional magnetic domain walls, European J. Appl. Math., 15 (2004), pp. 451-486.

[6] - Néel walls in low anisotropy symmetric double layers, SIAM J. Appl Math., 65 (2005), pp. 1726-1747. 
[7] — Structure of the Bloch wall in multilayers, Proc. R. Soc. Lond. Ser. A Math. Phys. Engr. Sci., 461 (2005), pp. 1911-1926.

[8] — Numerical micromagnetics: A review, Bol. Soc. Esp. Mat. Apl., 39 (2007), pp. 103-135.

[9] E. C. Gartland, JR. AND A. Ramage, Local stability and a renormalized Newton method for liquid crystal director modeling, tech. rep., University of Strathclyde, Department of Mathematics and Statistics, Research Report 2012 \#9, June 2012.

[10] — Local stability assessment and optimal preconditioning for liquid crystal director modeling, 2014. In preparation.

[11] P. E. Gill, W. Murray, and M. H. Wright, Practical Optimization, Academic Press, London, 1981.

[12] R. Glowinski and P. L. Tallec, Augmented Lagrangian and Operator-Splitting Methods in Nonlinear Mechanics, vol. 9 of Studies in Applied Mathematics, SIAM, Philadelphia, 1989.

[13] R. HARdt, D. Kinderlehrer, AND F.-H. Lin, Existence and partial regularity of static liquid crystal configurations, Commun. Math. Phys., 105 (1986), pp. 547-570.

[14] Q. Hu, X.-C. TAI, AND R. Winther, A saddle point approach to the computation of harmonic maps, SIAM J. Numer. Anal., 47 (2009), pp. 1500-1523.

[15] C. T. Kelley, Iterative Methods for Linear and Nonlinear Equations, SIAM, Philadelphia, 1995.

[16] M. KružíK And A. Prohl, Recent developments in the modeling, analysis, and numerics of ferromagnetism, SIAM Review, 48 (2006), pp. 439-483.

[17] S.-Y. Lin AND M. Luskin, Relaxation methods for liquid crystal problems, SIAM J. Numer. Anal., 26 (1989), pp. 1310-1324.

[18] J. Nocedal and S. J. Wright, Numerical Optimization, Springer-Verlag, New York, 1999.

[19] J. M. Ortega And W. C. Rheinboldt, Iterative Solution of Nonlinear Equations in Several Variables, Academic Press, Inc., Orlando, 1970.

[20] A. Prohl, Computational Micromagnetism, Teubner, Stuttgart, 2001.

[21] A. Ramage and E. C. Gartland, JR., A preconditioned nullspace method for liquid crystal director modeling, SIAM J. Sci. Comput., 35 (2013), pp. B226-B247.

[22] I. W. Stewart, The Static and Dynamic Continuum Theory of Liquid Crystals: A Mathematical Introduction, Taylor and Francis, London, 2004.

[23] E. G. Virga, Variational Theories for Liquid Crystals, Chapman \& Hall, London, 1994. 